\title{
Multiparameter singular integrals and maximal operators along flat surfaces
}

\author{
Yong-Kum Cho, Sunggeum Hong, Joonil Kim \\ and Chan Woo Yang
}

\begin{abstract}
We study double Hilbert transforms and maximal functions along surfaces of the form $\left(t_{1}, t_{2}, \gamma_{1}\left(t_{1}\right) \gamma_{2}\left(t_{2}\right)\right)$. The $L^{p}\left(\mathbb{R}^{3}\right)$ boundedness of the maximal operator is obtained if each $\gamma_{i}$ is a convex increasing and $\gamma_{i}(0)=0$. The double Hilbert transform is bounded in $L^{p}\left(\mathbb{R}^{3}\right)$ if both $\gamma_{i}$ 's above are extended as even functions. If $\gamma_{1}$ is odd, then we need an additional comparability condition on $\gamma_{2}$. This result is extended to higher dimensions and the general hyper-surfaces of the form $\left(t_{1}, \ldots, t_{n}, \Gamma\left(t_{1}, \ldots, t_{n}\right)\right)$ on $\mathbb{R}^{n+1}$.
\end{abstract}

\section{Introduction}

As an extension of Calderón-Zygmund theory, singular Radon transforms and maximal functions along the appropriate submnifolds have been intensively studied. Their $L^{p}$ boundedness is determined by certain geometric conditions that are described in numerous ways. In [5], Nagel, Christ, Stein, Wainger have shown the equivalence of those finite type conditions in the very general setting. An interesting problem is to establish the $L^{p}$ theory for flat manifolds, which are in lack of the finite type condition. We have fairly good understanding of the flat curves of the form $(t, \gamma(t))$ in $[1,4,6,8]$, even though appropriate extensions for general flat surfaces are not well known. In $[4,6]$, the $L^{p}$ boundedness of the maximal operators and singular integrals associated with the convex curves of the form $(t, \gamma(t))$ where $\gamma(0)=\gamma^{\prime}(0)=0$ has been obtained under the doubling type condition of $\gamma^{\prime}$, that is, $\gamma^{\prime}(C t) \geq 2 \gamma^{\prime}(t)$ for all $t>0$ with some $C>0$. This condition is a

2000 Mathematics Subject Classification: Primary 42B20, 42B25.

Keywords: Singular Radon transform, multiple Hilbert transform, flat surface. 
necessary and sufficient condition for $L^{p}$ boundedness of singular integrals along even curves. Singular integral for odd $\gamma$ was proved to be bounded in $L^{2}$ if and only if for some $C>0, h(C t) \geq 2 h(t)$ for all $t>0$ where $h(t)=t \gamma^{\prime}(t)-\gamma(t)$ in [8]. The $L^{p}$ boundedness with $p \neq 2$ was also obtained in [1] if there is $\epsilon>0$ such that $h^{\prime}(t)>\epsilon h(t) / t$ for all $t>0$. Singular integrals associated with higher dimensional flat submanifold of the form $\left\{(t, \gamma(|t|)): t \in \mathbb{R}^{n}\right\}$ have been considered in $[7,11,12,14]$.

It would be natural to ask how one can set up an appropriate geometric condition for the $L^{p}$-boundedness of the multi-parameter singular Radon transforms and related maximal operators. Nagel and Wainger in [9] proved the $L^{2}$ boundedness of multiple Hilbert transforms associated with $k$-dimensional surfaces in $\mathbb{R}^{n}$, which have a certain dilation condition. Ricci and Stein in [10] established the $n$-parameter theory by using a general family of multi-parameter dilations. Their results combined with lifting argument in [13, Chapter 11] imply the $L^{p}$ boundedness of the maximal operators associated with finite type surfaces as well as polynomial surfaces. In [3], Carbery, Wainger and Wright have found the necessary and sufficient condition for the $L^{p}\left(\mathbb{R}^{3}\right)$ boundedness of the double Hilbert transforms associated with polynomial surfaces. It is also known that only finite type condition does not imply $L^{p}$ boundedness of multiple Hilbert transform (see Remark 4 and $[2])$.

Next interesting question is what conditions determine the $L^{p}$ boundedness of the multi-parameter maximal operators and singular integrals along flat surfaces without finite type conditions. The purpose of this paper is to establish appropriate sufficient conditions for the $L^{p}$ boundedness. We begin with considering model surfaces of the form $\left\{(t, \Gamma(t)): t \in \mathbb{R}^{2}\right\}$ where $\Gamma\left(t_{1}, t_{2}\right)=\gamma_{1}\left(t_{1}\right) \gamma_{2}\left(t_{2}\right)$ and $\gamma_{i}$ is a convex function such that $\gamma_{i}(0)=\gamma_{i}^{\prime}(0)=0$. Interestingly it turns out that we do not need any additional condition such as the doubling type condition of $\gamma^{\prime}$ and $h$ in any direction $t_{1}$ or $t_{2}$. However as we extend this to general $\Gamma$, we come up with a curvature condition which is close to infinitesimal doubling condition of curve theory in [1].

\subsection{Theorems of a model case}

We shall consider the multiple Hilbert transforms and maximal operators associated with the hypersurfaces in $\mathbb{R}^{3}$ of the form $\left\{\left(t_{1}, t_{2}, \gamma_{1}\left(t_{1}\right) \gamma_{2}\left(t_{2}\right)\right)\right\}$ where $\gamma_{i}:[0, \infty) \mapsto \mathbb{R}$ be a $C^{2}$ convex function for each $i=1,2$. We define a strong maximal function $M f$ along this surface

$$
M f(x)=\sup _{r_{1}, r_{2}>0} \frac{1}{r_{1} r_{2}} \int_{0}^{r_{1}} \int_{0}^{r_{2}}\left|f\left(x_{1}-t_{1}, x_{2}-t_{2}, x_{3}-\gamma_{1}\left(t_{1}\right) \gamma_{2}\left(t_{2}\right)\right)\right| d t_{1} d t_{2} .
$$


If each $\gamma_{i} \quad(i=1,2)$ is suitably extended on $(-\infty, \infty)$, then the double Hilbert transform along this extended surface is defined by

$$
H f(x)=\lim _{\epsilon_{1}, \epsilon_{2} \rightarrow 0} \iint_{I_{\epsilon_{1}} \times I_{\epsilon_{2}}} f\left(x_{1}-t_{1}, x_{2}-t_{2}, x_{3}-\gamma_{1}\left(t_{1}\right) \gamma_{2}\left(t_{2}\right)\right) \frac{d t_{1}}{t_{1}} \frac{d t_{2}}{t_{2}},
$$

where $I_{\epsilon}=\left\{s: \epsilon<s<\frac{1}{\epsilon}\right\}$. The $L^{p}$ boundedness of the maximal operator $M$ can be shown under the only convexity assumption for each $\gamma_{i}$. Also we prove the similar result for the double Hilbert transform $H$ when each $\gamma_{i}$ is extended as an even function.

Theorem 1. Suppose that $\gamma_{i}$ is a convex function on $[0, \infty)$ such that $\gamma_{i}(0)=$ $\gamma_{i}^{\prime}(0)=0$ for each $i=1,2$. Then $M$ is bounded on $L^{p}\left(\mathbb{R}^{3}\right)$ for $1<p \leq \infty$.

Theorem 2. Suppose that $\gamma_{i}$ is a convex function on $[0, \infty)$ such that $\gamma_{i}(0)=$ $\gamma_{i}^{\prime}(0)=0$ for each $i=1,2$. If each $\gamma_{i}$ is extended as an even function in $(-\infty, \infty)$, then $H$ is bounded on $L^{p}\left(\mathbb{R}^{3}\right)$ for $1<p<\infty$.

Theorem 3. Suppose that $\gamma_{i}$ is a convex function on $[0, \infty)$ such that $\gamma_{i}(0)=$ $\gamma_{i}^{\prime}(0)=0$ for each $i=1,2$. Let $\gamma_{1}$ be extended as an odd function and $\gamma_{2}$ be extended as an even function in $(-\infty, \infty)$. If $\sup _{t \geq 0}\left|\frac{\gamma_{2}(2 t)}{\gamma_{2}(t)}\right|$ is bounded, then $H$ is bounded on $L^{p}\left(\mathbb{R}^{3}\right)$ for $1<p<\infty$.

Remark 1. (i) For the case that both of $\gamma_{i}$ 's are odd monomials, it is known that the $L^{p}$ norm of $H$ is unbounded for any $1<p<\infty$. Thus we are not concerned with the case that both of $\gamma_{i}$ 's are odd functions.

(ii) Examples. The hypotheses of Theorems 1 and 2 are satisfied by a large class of flat surfaces, of course, including the surface of the form

$$
\left(t_{1}, t_{2}, e^{-1 / t_{1}} e^{-1 / t_{2}}\right),
$$

near the origin. Our assumption that $\gamma_{i} \in C^{2}$ can be removed by using smooth approximation $\gamma_{i}^{\epsilon}$ so as to include any piecewise linear convex function $\gamma_{i}$ (see the details in Remark 3 at the end of Section 3.) Theorem 3 covers the surface which is flat along one direction such as $\left(t_{1}, t_{2}, e^{-1 / t_{1}} t_{2}^{2 m}\right)$ where $m$ is a positive integer. In Sections 5-7, we extend Theorems 1 through 3 to more general hyper-surfaces of the form

$$
\left(t_{1}, \ldots, t_{n}, \Gamma\left(t_{1}, \ldots, t_{n}\right)\right)
$$

We note that $\Gamma$ needs not be a tensor product. A model example will be

$$
\left(t_{1}, \ldots, t_{n}, \exp \left(-\frac{1}{t_{1}^{m_{1}} \cdots t_{n}^{m_{n}}}\right)\right)
$$

where $m_{i}$ is a positive integer.

(iii) If $\Gamma\left(t_{1}, t_{2}\right)$ is given as $\gamma_{1}\left(t_{1}\right)+\gamma_{2}\left(t_{2}\right)$, then we can easily check that our two parameter problem reduces to one parameter case. So we need the doubling properties of $\gamma_{i}^{\prime}\left(t_{i}\right)$ or $h_{i}\left(t_{i}\right)=\gamma_{i}^{\prime}\left(t_{i}\right) t_{i}-\gamma_{i}\left(t_{i}\right)$ as in $[4,8]$. 


\subsection{Scheme of proofs}

We briefly explain the main idea for the proof of Theorems 1 through 3 . Choose a nonnegative function $\varphi \in C_{0}^{\infty}\left[\frac{1}{2}, 1\right]$ such that $\int \varphi d t=1$. It suffices to deal with the maximal function

$$
\mathfrak{M} f(x)=\sup _{J \in \mathbb{Z}^{2}}\left|M_{J}^{1,2} * f(x)\right|,
$$

where the Fourier transform of the measure $M_{J}^{1,2}$ is given by

$$
\widehat{M_{J}^{1,2}}(\xi)=\iint e^{i\left[\xi_{1} 2^{j_{1}} t_{1}+\xi_{2} 2^{j_{2}} t_{2}+\xi_{3} \gamma_{1}\left(2^{j_{1}} t_{1}\right) \gamma_{2}\left(2^{j_{2}} t_{2}\right)\right]} \varphi\left(t_{1}\right) \varphi\left(t_{2}\right) d t_{1} d t_{2}
$$

for $J=\left(j_{1}, j_{2}\right) \in \mathbb{Z}^{2}$ and $\xi=\left(\xi_{1}, \xi_{2}, \xi_{3}\right) \in \mathbb{R}^{3}$. First we decompose the operators into two parts; local and global parts based on the size of the frequency variables $\xi_{1}$ and $\xi_{2}$.

To treat the global part we make use of the decay property of $\widehat{M_{J}^{1,2}}(\xi)$ in $\left|2^{j_{1}} \xi_{1}\right|$ and $\left|2^{j_{2}} \xi_{2}\right|$. The decay estimate can be successfully obtained from the fact that $\gamma_{1}$ and $\gamma_{2}$ help each other when one of them changes slowly. In the region where $\gamma_{1}$ changes slowly, one can make a measure estimate in $t_{2}$ variable by using the convexity of $\gamma_{2}$. We shall show that this kind of multidimensional Van der Corput type lemma (Lemma 1 in the next section) can also be extended to more general surfaces in higher dimensional Euclidean spaces in Section 6 .

For the local part estimate, we subtract our original operators by operators associated with surfaces of the form $\left(0, t_{2}, \gamma_{1}\left(t_{1}\right) \gamma_{2}\left(t_{2}\right)\right)$ and use the mean value theorem to deal with the difference. It remains to take into account of the maximal function or the double Hilbert transform along this surface $\left(0, t_{2}, \gamma_{1}\left(t_{1}\right) \gamma_{2}\left(t_{2}\right)\right)$. We will see in Lemmas 2-4 that the corresponding maximal function is reduced to the composition of Hardy-Littlewood maximal functions. The corresponding double Hilbert transform vanishes when both $\gamma_{i}$ 's are even by using the cancellation property of the measure $\frac{d t_{1}}{t_{1}}$, but we do not have such cancellation when $\gamma_{1}$ is odd and $\gamma_{2}$ is even. For this case we need to control the size of the frequency variables $\xi_{2}$ and $\xi_{3}$ instead of $\xi_{2}$ and $\xi_{1}$. It is technically difficult to obtain the decay estimate for $\xi_{3}$ without any restrictions on the size of derivatives of $\gamma_{1}\left(t_{1}\right) \gamma_{2}\left(t_{2}\right)$. We are able to obtain the $L^{p}$ estimates under the condition such as $\left|\gamma_{2}\left(2^{j_{2}}\right)\right| \approx\left|\gamma_{2}\left(2^{j_{2}+1}\right)\right|$ in Theorem 3. It would be interesting to remove this kind of comparability condition.

This paper consists of two parts. In the first part (Sections 2-4), we consider the model case. In the second parts (Sections 5-7), we treat the general $n$ dimensional surface in $\mathbb{R}^{n+1}$ where $\Gamma$ need not be a tensor product 
form. In Section 2, we prove Van der Corput type lemma and show how the maximal operator along $\left(0, t_{2}, \gamma_{1}\left(t_{1}\right) \gamma_{2}\left(t_{2}\right)\right)$ is reduced to Hardy-Littlewood maximal function. In Sections 3 and 4, we give the proofs of Theorems 1-3. In Sections 5, from the observation of the previous proofs we formulate an appropriate geometric conditions corresponding to general $\Gamma$ and states Theorems 4-6. In Sections 6-7, we prove them.

We shall use the notation $A \lesssim B$ when $A \leq C B$ with a constant $C>0$.

\section{Preliminary Lemmas}

\subsection{Global estimates (Van der Corput type lemma)}

Lemma 1. Suppose that $\gamma_{k}$ is a convex function on $[0, \infty)$ such that $\gamma_{k}(0)=$ $\gamma_{k}^{\prime}(0)=0$ for each $k=1,2$. Then we have

$$
\left|\widehat{M_{J}^{1,2}}(\xi)\right| \lesssim \min \left\{\frac{1}{\left|2^{j_{1}} \xi_{1}\right|^{1 / 2}}, \frac{1}{\left|2^{j_{2}} \xi_{2}\right|^{1 / 2}}\right\}
$$

Proof. By symmetry it suffices to show that $\left|\widehat{M_{J}^{1,2}}(\xi)\right|$ is bounded by $\frac{1}{\left|2^{j_{1}} \xi_{1}\right|^{1 / 2}}$. We may assume that $\left|2^{j_{1}} \xi_{1}\right|>10^{3}$. Let $\chi$ be a smooth even function supported in $[-4,-1 / 4] \cup[1 / 4,4]$ and $\chi \equiv 1$ on $[-2,-1 / 2] \cup[1 / 2,2]$. Then we write for fixed $J=\left(j_{1}, j_{2}\right)$

$$
\widehat{M_{J}^{1,2}}(\xi)=\sum_{n} M_{n}(\xi)
$$

where

$$
\begin{aligned}
M_{n}(\xi)= & \iint e^{i\left[\xi_{1} 2^{j_{1}} t_{1}+\xi_{2} 2^{j_{2}} t_{2}+\xi_{3} \gamma_{1}\left(2^{j_{1}} t_{1}\right) \gamma_{2}\left(2^{j_{2}} t_{2}\right)\right]} \\
& \times \chi\left(\frac{2^{j_{1}}\left(\xi_{1}+\xi_{3} \gamma_{1}^{\prime}\left(2^{j_{1}} t_{1}\right) \gamma_{2}\left(2^{j_{2}} t_{2}\right)\right)}{2^{n}}\right) \varphi\left(t_{1}\right) \varphi\left(t_{2}\right) d t_{1} d t_{2} .
\end{aligned}
$$

If $2^{n}>\left|2^{j_{1}} \xi_{1}\right|^{1 / 2}$, let us apply integration by parts with respect to $t_{1}$ variable for $M_{n}(\xi)$, then

$$
\begin{aligned}
\left|M_{n}(\xi)\right| & \lesssim \iint\left|\frac{\partial}{\partial t_{1}}\left(\frac{\chi\left(\frac{2^{j_{1}}\left(\xi_{1}+\xi_{3} \gamma_{1}^{\prime}\left(2^{j_{1}} t_{1}\right) \gamma_{2}\left(2^{j_{2}} t_{2}\right)\right)}{2^{n}}\right) \varphi\left(t_{1}\right) \varphi\left(t_{2}\right)}{2^{j_{1}}\left(\xi_{1}+\xi_{3} \gamma_{1}^{\prime}\left(2^{j_{1}} t_{1}\right) \gamma_{2}\left(2^{j_{2}} t_{2}\right)\right)}\right)\right| d t_{1} d t_{2} \\
& \lesssim 2^{-n}
\end{aligned}
$$

where the second inequality follows by using the monotonicity of the function $t_{1} \mapsto 1 /\left(\xi_{1}+\gamma_{1}^{\prime}\left(2^{j_{1}} t_{1}\right) \gamma_{2}\left(2^{j_{2}} t_{2}\right)\right)$ with each fixed $t_{2}$. Thus we see that

$$
\sum_{2^{n}>\left|2^{j_{1}} \xi_{1}\right|^{1 / 2}}\left|M_{n}(\xi)\right| \lesssim\left|2^{j_{1}} \xi_{1}\right|^{-1 / 2}
$$


To consider the case where $2^{n} \leq\left|2^{j_{1}} \xi_{1}\right|^{1 / 2}$, we first observe that the convexity of the map $t_{2} \mapsto \gamma_{2}\left(t_{2}\right)$ with $\gamma_{2}(0)=\gamma_{2}^{\prime}(0)=0$ yields that

$$
\gamma_{2}^{\prime}\left(t_{2}\right) t_{2} \geq \gamma_{2}\left(t_{2}\right) \text { for all } t_{2} \geq 0
$$

which implies

$$
\gamma_{1}^{\prime}\left(2^{j_{1}} t_{1}\right) \gamma_{2}^{\prime}\left(2^{j_{2}} t_{2}\right) 2^{j_{2}} t_{2} \geq \gamma_{1}^{\prime}\left(2^{j_{1}} t_{1}\right) \gamma_{2}\left(2^{j_{2}} t_{2}\right)
$$

By using the support condition such that

$$
\left|2^{j_{1}}\left(\xi_{1}+\xi_{3} \gamma_{1}^{\prime}\left(2^{j_{1}} t_{1}\right) \gamma_{2}\left(2^{j_{2}} t_{2}\right)\right)\right|<5\left|2^{j_{1}} \xi_{1}\right|^{1 / 2}<\left|2^{j_{1}} \xi_{1}\right| / 10,
$$

we have

$$
\left.\mid \xi_{3} \gamma_{1}^{\prime}\left(2^{j_{1}} t_{1}\right) \gamma_{2}\left(2^{j_{2}} t_{2}\right)\right)|\approx| \xi_{1} \mid
$$

From (2.2) and (2.3), we obtain

$$
\left|\xi_{3} 2^{j_{1}} \gamma_{1}^{\prime}\left(2^{j_{1}} t_{1}\right) \gamma_{2}^{\prime}\left(2^{j_{2}} t_{2}\right) 2^{j_{2}}\right| \geq\left|\xi_{3} 2^{j_{1}} \gamma_{1}^{\prime}\left(2^{j_{1}} t_{1}\right) \gamma_{2}\left(2^{j_{2}} t_{2}\right)\right| \approx\left|2^{j_{1}} \xi_{1}\right| .
$$

By using (2.4), we estimate the measure of the domain of the integral in $d t_{2}$ with $t_{1}$ fixed to obtain

$$
\begin{aligned}
\left|M_{n}(\xi)\right| & \lesssim \iint\left|\chi\left(\frac{2^{j_{1}}\left(\xi_{1}+\xi_{3} \gamma_{1}^{\prime}\left(2^{j_{1}} t_{1}\right) \gamma_{2}\left(2^{j_{2}} t_{2}\right)\right)}{2^{n}}\right)\right| \varphi\left(t_{1}\right) \varphi\left(t_{2}\right) d t_{1} d t_{2} \\
& \lesssim \frac{2^{n}}{2^{j_{1}}\left|\xi_{1}\right|} .
\end{aligned}
$$

Thus we have

$$
\sum_{2^{n} \leq\left|2^{j_{1}} \xi_{1}\right|^{1 / 2}}\left|M_{n}(\xi)\right| \lesssim\left|2^{j_{1}} \xi_{1}\right|^{-1 / 2}
$$

By (2.1) and (2.5) we complete the proof. Note that our proof is based on the curvature condition (2.2). In order to obtain the decay factor $\left|2^{j_{2}} \xi_{2}\right|^{1 / 2}$, we use the corresponding condition

$$
\gamma_{2}^{\prime}\left(2^{j_{2}} t_{2}\right) \gamma_{1}^{\prime}\left(2^{j_{1}} t_{1}\right) 2^{j_{1}} t_{1} \geq \gamma_{2}^{\prime}\left(2^{j_{2}} t_{2}\right) \gamma_{1}\left(2^{j_{1}} t_{1}\right)
$$

which follows from the convexity condition of $\gamma_{1}$ and $\gamma_{1}(0)=\gamma_{1}^{\prime}(0)=0$.

Remark 2. We define the measure $M_{J}^{1}$ and $M_{J}^{2}$ so that their Fourier transforms are given by

$$
\begin{aligned}
& \widehat{M_{J}^{1}}(\xi)=\iint e^{i\left[\xi_{1} 2^{j_{1}} t_{1}+\xi_{3} \gamma_{1}\left(2^{j_{1}} t_{1}\right) \gamma_{2}\left(2^{j_{2}} t_{2}\right)\right]} \varphi\left(t_{1}\right) \varphi\left(t_{2}\right) d t_{1} d t_{2}, \\
& \widehat{M_{J}^{2}}(\xi)=\iint e^{i\left[\xi_{2} 2^{j_{2}} t_{2}+\xi_{3} \gamma_{1}\left(2^{j_{1}} t_{1}\right) \gamma_{2}\left(2^{j_{2}} t_{2}\right)\right]} \varphi\left(t_{1}\right) \varphi\left(t_{2}\right) d t_{1} d t_{2} .
\end{aligned}
$$

Then by using the same argument as above we obtain that for each $\nu=1,2$,

$$
\left|\widehat{M_{J}^{\nu}}(\xi)\right| \lesssim \frac{1}{\left|2^{j_{\nu}} \xi_{\nu}\right|^{1 / 2}}
$$




\subsection{Local estimates (reduction to strong maximal function)}

For local estimates we reduce a certain maximal operator into a composition of Hardy-Littlewood maximal functions. For this purpose we need the following observation as in [6]:

Lemma 2. Let $\gamma:[0, \infty) \mapsto \mathbb{R}$ be a convex increasing function. We define the maximal operator $M_{\gamma}$ by

$$
M_{\gamma} f(x)=\sup _{r>0} \frac{1}{r} \int_{0}^{r}|f(x-\gamma(s))| d s .
$$

Then for each $x \in \mathbb{R}$, we have

$$
M_{\gamma} f(x) \leq 2 M_{H L} f(x-\gamma(0))
$$

where $M_{H L}$ is the Hardy-Littlewood maximal function.

Proof. Suppose that $\gamma^{\prime}(t)>0$ for all $t>0$. By using change of variable $t=\gamma(s)$, we write

$$
\frac{1}{r} \int_{0}^{r}|f(x-\gamma(s))| d s=\int|f(x-t)| \Omega(t) d t
$$

where

$$
\Omega(t)=\chi_{[\gamma(0), \gamma(r)]}(t) \frac{1}{\gamma^{\prime}\left(\gamma^{-1}(t)\right) r} .
$$

In view of the convexity of $\gamma, \Omega$ is a positive bounded decreasing function and by change of variable $s=\gamma^{-1}(t)$, it is easy to see that $\int \Omega(t) d t=1$. Thus we can approximate $\Omega$ by using a step function $S_{N}$ of the form

$$
S_{N}(t)=\sum_{j=1}^{N} a_{j} \chi_{\left[\gamma(0), \gamma(0)+b_{j}\right]}(t)
$$

such that $0<b_{1}<b_{2}<\cdots<b_{N}<\gamma(r)-\gamma(0), \quad a_{j} \geq 0$, which satisfies

$$
\begin{aligned}
& S_{N}(t) \nearrow \Omega(t) \quad \text { as } N \rightarrow \infty \text { for all } t \in \mathbb{R}, \\
& \text { and } \int S_{N}(t) d t=\sum_{j=1}^{N} a_{j} b_{j} \leq 1 .
\end{aligned}
$$


We therefore have

$$
\begin{aligned}
\int|f(x-t)| \Omega(t) d t & =\lim _{N \rightarrow \infty} \int|f(x-t)| S_{N}(t) d t \\
& =\lim _{N \rightarrow \infty} \sum_{j=1}^{N} a_{j} b_{j}\left(\frac{1}{b_{j}} \int_{\gamma(0)}^{\gamma(0)+b_{j}}|f(x-t)| d t\right) \\
& \leq \lim _{N \rightarrow \infty} \sum_{j=1}^{N} a_{j} b_{j} M_{H L} f(x-\gamma(0)) \\
& \leq M_{H L} f(x-\gamma(0)) .
\end{aligned}
$$

Suppose that $[0, a]=\left\{t: \gamma^{\prime}(t)=0\right\}$ with some $a>0$. Then it suffices to consider $a<r$. Since $\gamma(t)=\gamma(0)$ on $[0, a]$, we can write

$$
\begin{aligned}
\frac{1}{r} \int_{0}^{r}|f(x-\gamma(s))| d s & =\frac{1}{r} \int_{0}^{a}|f(x-\gamma(0))| d s+\frac{1}{r} \int_{a}^{r}|f(x-\gamma(s))| d s \\
& \leq|f(x-\gamma(0))|+M_{H L} f(x-\gamma(0)) \\
& \leq 2 M_{H L} f(x-\gamma(0))
\end{aligned}
$$

where the first inequality above is shown by using the approximation of step functions as in the previous argument.

Let $\Gamma: \mathbb{R}^{n} \rightarrow \mathbb{R}$ be a function of $n$ variables. We define a multi-parameter maximal function associated with $\Gamma$ by

$$
\mathcal{M}_{\Gamma}^{n} f\left(x, x_{n+1}\right)=\sup _{r_{1}, \ldots, r_{n}>0} \frac{1}{\prod_{i=1}^{n} r_{i}} \int_{\otimes_{i=1}^{n}\left[0, r_{i}\right]}\left|f\left(x-t, x_{n+1}-\Gamma(t)\right)\right| d t,
$$

where $x=\left(x_{1}, \ldots, x_{n}\right)$. In dealing with this maximal operator, we need to consider a maximal function for each $D \subset\{1, \ldots, n\}$,

$$
\mathcal{S}_{\Gamma}^{n, D} f\left(x, x_{n+1}\right)=\sup _{r_{1}, \ldots, r_{n}>0} \frac{1}{\prod_{i=1}^{n} r_{i}} \int_{\otimes_{i=1}^{n}\left[0, r_{i}\right]}\left|f\left(x-\hat{t}_{D}, x_{n+1}-\Gamma(t)\right)\right| d t,
$$

where $x=\left(x_{1}, \ldots, x_{n}\right)$ and $\hat{t}_{D}=t-\sum_{\nu \in D}\left\langle t, \mathbf{e}_{\nu}\right\rangle \mathbf{e}_{\nu}$ is a vector given by omitting all entries in $\nu$-th coordinates of $t$ where $\nu \in D$. By repeated application of the previous lemma, we obtain the following higher dimensional result.

Lemma 3. Suppose that $t_{\nu} \mapsto \Gamma(t)$ is a convex increasing function for each fixed $\hat{t}_{\{\nu\}}$ where $\nu \in D$. Then

$$
\begin{aligned}
& \mathcal{S}_{\Gamma}^{n, D} f\left(x, x_{n+1}\right) \leq \\
& \quad \leq \sup _{r_{i}>0 \text { with } i \notin D} \frac{2^{\operatorname{card}(D)}}{\prod_{i \notin D} r_{i}} \int_{\bigotimes_{i \notin D}\left[0, r_{i}\right]} M_{S}^{D} f\left(x-\hat{t}_{D}, x_{n+1}-\Gamma\left(\hat{t}_{D}\right)\right) d \hat{t}_{D},
\end{aligned}
$$

where $M_{S}^{D}$ is the strong maximal operator with respect to the $\nu$ 's coordinates with $\nu \in D$ and $\operatorname{card}(D)$ is the number of elements in the set $D$. 
From Lemma 3, we obtain the stronger result for the case $\Gamma\left(\hat{t}_{D}\right)=0$.

Lemma 4. Let $\Gamma(t)=\prod_{i=1}^{n} \gamma_{i}\left(t_{i}\right)$ and $\gamma_{i}$ be a convex function with $\gamma_{i}(0)=$ $\gamma_{i}^{\prime}(0)=0$. If $D \neq \emptyset$,

$$
\mathcal{S}_{\Gamma}^{n, D} f\left(x, x_{n+1}\right) \leq 2 M_{S} f\left(x, x_{n+1}\right)
$$

where $M_{S} f$ is the strong maximal function defined on $\mathbb{R}^{n+1}$.

\subsection{Littlewood-Paley type inequalities}

In order to sum all dyadic pieces and obtain $L^{p}$ estimates we need Littlewood-Paley type inequalities. Let us define dyadic decompositions of each frequency variable $\xi=\left(\xi_{1}, \ldots, \xi_{d}\right) \in \mathbb{R}^{d}$ by

$$
\widehat{L_{k}^{\nu}}(\xi)=\chi\left(2^{k} \xi_{\nu}\right)
$$

where $\nu=1, \ldots, d$, and $\chi(u)=\psi(u)-\psi(2 u)$ with $\psi$ a $C^{\infty}$ function such that $\psi=1$ on $[-1,1]$ and $\psi=0$ on $\mathbb{R} \backslash[-2,2]$. We also define for each $\nu$,

$$
\begin{aligned}
\widehat{P_{k}^{\nu}}(\xi) & =\psi\left(2^{k} \xi_{\nu}\right), \\
\widehat{\left(P_{k}^{\nu}\right)^{c}}(\xi) & =\psi^{c}\left(2^{k} \xi_{\nu}\right)=1-\psi\left(2^{k} \xi_{\nu}\right) .
\end{aligned}
$$

We write the following well known inequalities which will be used for the $L^{p}$ estimates of our maximum or the square sum of dyadic pieces.

Lemma 5. For $1<p<\infty$, we have for $J=\left(j_{1}, \ldots, j_{n}\right)$ and $\nu=1, \ldots, d$,

$$
\begin{aligned}
& \left\|\left(\sum_{J \in \mathbb{Z}^{n}}\left|L_{j_{1}}^{1} * \cdots * L_{j_{n}}^{n} * f\right|^{2}\right)^{\frac{1}{2}}\right\|_{L^{p}\left(\mathbb{R}^{d}\right)} \lesssim\|f\|_{L^{p}\left(\mathbb{R}^{d}\right)}, \\
& \sup _{k}|| P_{k}^{\nu}|* f(x)| \lesssim\left|M_{H L}^{\nu} f(x)\right|, \\
& \left\|\left(\left.\sum_{J \in \mathbb{Z}^{n}}\left|\sup _{k}\right|\left|P_{k}^{\nu}\right| * f_{J}\right|^{2}\right)^{\frac{1}{2}}\right\|_{L^{p}\left(\mathbb{R}^{d}\right)} \lesssim\left\|\left(\sum_{J \in \mathbb{Z}^{n}}\left|f_{J}\right|^{2}\right)^{\frac{1}{2}}\right\|_{L^{p}\left(\mathbb{R}^{d}\right)},
\end{aligned}
$$

where $M_{H L}^{\nu}$ is the Hardy-Littlewood maximal operator along the $\nu$-axis.

Proof. The first inequality follows by applying the multiparameter Marcinkiwicz multiplier theorem after switching to linear sums. The second inequality follows from the direct computation and the third follows from the vector valued inequality for the Hardy-Littlewood maximal function. 
Lemma 6. Suppose that $\sigma_{J}$ is a positive measure in $\mathbb{R}^{d}$ where $J$ is a multi index. And we assume that

$$
\left\|\sup _{J}\left|\sigma_{J} * f\right|\right\|_{L^{p_{0}\left(\mathbb{R}^{d}\right)}} \lesssim\|f\|_{L^{p_{0}\left(\mathbb{R}^{d}\right)}}, \quad \text { and } \quad\left\|\left|\sigma_{J} * f\right|\right\|_{L^{1}\left(\mathbb{R}^{d}\right)} \lesssim\|f\|_{L^{1}\left(\mathbb{R}^{d}\right)}
$$

for some $p_{0} \leq 2$. Then we have for $\frac{1}{p} \leq \frac{1}{2}\left(1+\frac{1}{p_{0}}\right)$,

$$
\left\|\left(\sum_{J}\left|\sigma_{J} * f_{J}\right|^{2}\right)^{\frac{1}{2}}\right\|_{L^{p}\left(\mathbb{R}^{d}\right)} \lesssim\left\|\left(\sum_{J}\left|f_{J}\right|^{2}\right)^{\frac{1}{2}}\right\|_{L^{p}\left(\mathbb{R}^{d}\right)} .
$$

Proof. Let $\mathfrak{f}=\left\{f_{J}\right\}$ be a vector valued function, and let $\mathfrak{R}$ be an operator defined by $\mathfrak{R} \mathfrak{f}=\left\{\sigma_{J} * f_{J}\right\}$. The hypotheses above imply that

$\|\mathfrak{R f}\|_{L^{p_{0}\left(l^{\infty}\left(\mathbb{R}^{d}\right)\right)}} \lesssim\|\mathfrak{f}\|_{L^{p_{0}\left(l^{\infty}\left(\mathbb{R}^{d}\right)\right)}}, \quad$ and $\quad\|\mathfrak{R} \mathfrak{f}\|_{L^{1}\left(l^{1}\left(\mathbb{R}^{d}\right)\right)} \lesssim\|\mathfrak{f}\|_{L^{1}\left(l^{1}\left(\mathbb{R}^{d}\right)\right)}$.

By the interpolation of the two vector valued spaces $L^{p_{0}}\left(l^{\infty}\left(\mathbb{R}^{d}\right)\right)$ and $L^{1}\left(l^{1}\left(\mathbb{R}^{d}\right)\right)$, we complete the proof.

\section{Proof of Theorem 1}

Now we are ready to prove Theorem 1 . We decompose $M_{J}^{1,2}$ such that

$$
M_{J}^{1,2}=M_{J}^{l o c}+M_{J}^{g l o}+M_{J}^{m i d}
$$

where

$$
\begin{aligned}
\widehat{M_{J}^{\text {loc }}}(\xi) & =\widehat{M_{J}^{1,2}}(\xi) \psi\left(2^{j_{1}} \xi_{1}\right) \psi\left(2^{j_{2}} \xi_{2}\right) \\
\widehat{M_{J}^{g l o}}(\xi) & =\widehat{M_{J}^{1,2}}(\xi) \psi^{c}\left(2^{j_{1}} \xi_{1}\right) \psi^{c}\left(2^{j_{2}} \xi_{2}\right) \\
\widehat{M_{J}^{\text {mid }}}(\xi) & =\widehat{M_{J}^{1,2}}(\xi) \psi\left(2^{j_{1}} \xi_{1}\right) \psi^{c}\left(2^{j_{2}} \xi_{2}\right)+\widehat{M_{J}^{1,2}}(\xi) \psi^{c}\left(2^{j_{1}} \xi_{1}\right) \psi\left(2^{j_{2}} \xi_{2}\right) .
\end{aligned}
$$

First we show that

$$
\left\|\sup _{J \in \mathbb{Z}^{2}}\left|M_{J}^{l o c} * f\right|\right\|_{L^{p}\left(\mathbb{R}^{3}\right)} \lesssim\|f\|_{L^{p}\left(\mathbb{R}^{3}\right)}
$$

Proof. For the proof we show that

$$
\begin{aligned}
& \left\|\sup _{J \in \mathbb{Z}^{2}}\left|\left(M_{J}^{1,2}-M_{J}^{1}-M_{J}^{2}+M_{J}^{0}\right) * P_{j_{1}}^{1} * P_{j_{2}}^{2} * f\right|\right\|_{L^{p}\left(\mathbb{R}^{3}\right)} \lesssim\|f\|_{L^{p}\left(\mathbb{R}^{3}\right)}, \\
& \left\|\sup _{J \in \mathbb{Z}^{2}}\left|M_{J}^{\nu} * P_{j_{1}}^{1} * P_{j_{2}}^{2} * f\right|\right\|_{L^{p}\left(\mathbb{R}^{3}\right)} \lesssim\|f\|_{L^{p}\left(\mathbb{R}^{3}\right)}, \text { for } \nu=0,1,2
\end{aligned}
$$


where $M_{J}^{1}$ and $M_{j}^{2}$ are defined in (2.7) and (2.8) respectively and $M_{J}^{0}$ is defined by

$$
\widehat{M_{J}^{0}}(\xi)=\iint e^{i \xi_{3} \gamma_{1}\left(2^{j_{1}} t_{1}\right) \gamma_{2}\left(2^{j_{2}} t_{2}\right)} \varphi\left(t_{1}\right) \varphi\left(t_{2}\right) d t_{1} d t_{2} .
$$

By Lemma 3, we see that for each $\nu=0,1,2$

$$
\left\|\sup _{J \in \mathbb{Z}^{2}}\left|M_{J}^{\nu} * f\right|\right\|_{L^{p}\left(\mathbb{R}^{3}\right)} \lesssim\|f\|_{L^{p}\left(\mathbb{R}^{3}\right)}
$$

which combined with (2.11) yields (3.2). For the estimate of (3.1), it suffices to show that

$$
\begin{aligned}
\|\left(\sum_{J} \mid\left(M_{J}^{1,2}-M_{J}^{1}-M_{J}^{2}+M_{J}^{0}\right) * P_{j_{1}}^{1} * P_{j_{2}}^{2} *\right. & \left.\left.L_{j_{1}-n_{1}}^{1} * L_{j_{2}-n_{2}}^{2} * f\right|^{2}\right)^{1 / 2} \|_{L^{p}} \\
& \lesssim 2^{-c\left(\left|n_{1}\right|+\left|n_{2}\right|\right)}\|f\|_{L^{p}}
\end{aligned}
$$

For the proof of (3.4) with $p=2$, we write the Fourier transform of the measure $M_{J}^{1,2}-M_{J}^{1}-M_{J}^{2}+M_{J}^{0}$ as

$$
\iint e^{i \xi_{3} \gamma_{1}\left(2^{j_{1}} t_{1}\right) \gamma_{2}\left(2^{j_{2}} t_{2}\right)}\left(e^{i \xi_{1} 2^{j_{1}} t_{1}}-1\right)\left(e^{i \xi_{2} 2^{j_{2}} t_{2}}-1\right) \varphi\left(t_{1}\right) \varphi\left(t_{2}\right) d t_{1} d t_{2} .
$$

By applying the mean value theorem and the support condition of the frequency part of the measure $P_{j_{1}}^{1} * P_{j_{2}}^{2} * L_{j_{1}-n_{1}}^{1} * L_{j_{2}-n_{2}}^{2}$, we obtain (3.4) with $p=2$. For the case $4 / 3<p<2$, we apply (2.10) and (2.12) in Lemma 5 and Lemma 6 combined with the previous $L^{2}$ result to obtain (3.4) with $c=0$. Finally the interpolation with $p=2$ in (3.4) gives the desired estimate for the range $4 / 3<p \leq 2$. Now we repeat this argument until we obtain the full range $1<p \leq 2$.

Next we prove that

$$
\left\|\sup _{J \in \mathbb{Z}^{2}}\left|M_{J}^{g l o} * f\right|\right\|_{L^{p}\left(\mathbb{R}^{3}\right)} \lesssim\|f\|_{L^{p}\left(\mathbb{R}^{3}\right)} .
$$

Proof. For the proof we show that

$$
\begin{aligned}
\|\left(\sum_{J} \mid\left(M_{J}^{1,2} *\left(P_{j_{1}}^{1}\right)^{c} *\left(P_{j_{2}}^{2}\right)^{c} * L_{j_{1}-n_{1}}^{1} *\right.\right. & \left.\left.L_{j_{2}-n_{2}}^{2} * f\right|^{2}\right)^{1 / 2} \|_{L^{p}} \\
& \lesssim 2^{-c\left(\left|n_{1}\right|+\left|n_{2}\right|\right)}\|f\|_{L^{p}}
\end{aligned}
$$

By applying Lemma 1 and the support condition of the frequency part of the measure $\left(P_{j_{1}}^{1}\right)^{c} *\left(P_{j_{2}}^{2}\right)^{c} * L_{j_{1}-n_{1}}^{1} * L_{j_{2}-n_{2}}^{2}$, we obtain (3.5) with $p=2$. For the case $1<p<2$, we apply the same bootstrap argument as above. 
We turn to the proof of

$$
\left\|\sup _{J \in \mathbb{Z}^{2}}\left|M_{J}^{m i d} * f\right|\right\|_{L^{p}\left(\mathbb{R}^{3}\right)} \lesssim\|f\|_{L^{p}\left(\mathbb{R}^{3}\right)} .
$$

It suffices to prove that

$$
\left\|\sup _{J \in \mathbb{Z}^{2}}\left|M_{J}^{1,2} * P_{j_{1}}^{1} *\left(P_{j_{2}}^{2}\right)^{c} * f\right|\right\|_{L^{p}\left(\mathbb{R}^{3}\right)} \lesssim\|f\|_{L^{p}\left(\mathbb{R}^{3}\right)} .
$$

Proof. For the proof we show that

$$
\begin{aligned}
& \left\|\sup _{J \in \mathbb{Z}^{2}}\left|\left(M_{J}^{1,2}-M_{J}^{2}\right) * P_{j_{1}}^{1} *\left(P_{j_{2}}^{2}\right)^{c} * f\right|\right\|_{L^{p}\left(\mathbb{R}^{3}\right)} \lesssim\|f\|_{L^{p}\left(\mathbb{R}^{3}\right)}, \\
& \left\|\sup _{J \in \mathbb{Z}^{2}}\left|M_{J}^{2} * P_{j_{1}}^{1} *\left(P_{j_{2}}^{2}\right)^{c} * f\right|\right\|_{L^{p}\left(\mathbb{R}^{3}\right)} \lesssim\|f\|_{L^{p}\left(\mathbb{R}^{3}\right)},
\end{aligned}
$$

where (3.7) follows from Lemma 4 and (2.11) in Lemma 5. For the estimate of (3.6), it suffices to show that

$$
\begin{aligned}
\|\left(\sum_{J} \mid\left(M_{J}^{1,2}-M_{J}^{2}\right) * P_{j_{1}}^{1} *\left(P_{j_{2}}^{2}\right)^{c} * L_{j_{1}-n_{1}}^{1} *\right. & \left.\left.L_{j_{2}-n_{2}}^{2} * f\right|^{2}\right)^{1 / 2} \|_{L^{p}} \\
& \lesssim 2^{-c\left(\left|n_{1}\right|+\left|n_{2}\right|\right)}\|f\|_{L^{p}}
\end{aligned}
$$

For the proof of (3.8) with $p=2$, we write the Fourier transform of the measure $M_{J}^{1,2}-M_{J}^{2}$ as

$$
\iint e^{i\left[\xi_{2} 2^{j_{2}} t_{2}+\xi_{3} \gamma_{1}\left(2^{j_{1}} t_{1}\right) \gamma_{2}\left(2^{j_{2}} t_{2}\right)\right]}\left(e^{i \xi_{1} 2^{j_{1}} t_{1}}-1\right) \varphi\left(t_{1}\right) \varphi\left(t_{2}\right) d t_{1} d t_{2}
$$

By applying the mean value theorem above and Lemma 1 and (2.9) and the support condition of the frequency part of the measure

$$
P_{j_{1}}^{1} *\left(P_{j_{2}}^{2}\right)^{c} * L_{j_{1}-n_{1}}^{1} * L_{j_{2}-n_{2}}^{2},
$$

we obtain (3.8) with $p=2$. The case $p \neq 2$ is obtained similarly as above.

Remark 3. The main assumption that $\gamma_{i} \in C^{2}$ can be removed. The condition such as $\gamma_{i}^{\prime \prime} \geq 0$ is used for performing integration by parts in proving Lemma 1. However we do not use the size of $\gamma_{i}^{\prime \prime}$. This enables us to repeat our previous proof with the curve replaced by the smooth approximated curve $\gamma_{i}^{\epsilon}(t)=\psi_{\epsilon} * \gamma_{i}(t)$ where $\psi$ is a nonegative $C_{0}^{\infty}$ function with the support near the origin such that

$$
\int \psi(t) d t=1 \quad \text { and } \quad \psi_{\epsilon}(t)=\frac{1}{\epsilon} \psi\left(\frac{t}{\epsilon}\right) .
$$




\section{Proofs of Theorems 2 and 3}

We decompose $H_{J}^{1,2}$ such that

$$
H_{J}^{1,2}=H_{J}^{l o c}+H_{J}^{g l o}+H_{J}^{\text {mid }}
$$

where

$$
\begin{aligned}
\widehat{H_{J}^{l o c}}(\xi) & =\widehat{H_{J}^{1,2}}(\xi) \psi\left(2^{j_{1}} \xi_{1}\right) \psi\left(2^{j_{2}} \xi_{2}\right), \\
\widehat{H_{J}^{g l o}}(\xi) & =\widehat{H_{J}^{1,2}}(\xi) \psi^{c}\left(2^{j_{1}} \xi_{1}\right) \psi^{c}\left(2^{j_{2}} \xi_{2}\right), \\
\widehat{H_{J}^{\text {mid }}}(\xi) & =\widehat{H_{J}^{1,2}}(\xi) \psi\left(2^{j_{1}} \xi_{1}\right) \psi^{c}\left(2^{j_{2}} \xi_{2}\right)+\widehat{H_{J}^{1,2}}(\xi) \psi^{c}\left(2^{j_{1}} \xi_{1}\right) \psi\left(2^{j_{2}} \xi_{2}\right) .
\end{aligned}
$$

We show that

$$
\begin{aligned}
\left\|\sum_{J \in \mathbb{Z}^{2}} H_{J}^{l o c} * f\right\|_{L^{p}\left(\mathbb{R}^{3}\right)} & \lesssim\|f\|_{L^{p}\left(\mathbb{R}^{3}\right)}, \\
\left\|\sum_{J \in \mathbb{Z}^{2}} H_{J}^{g l o} * f\right\|_{L^{p}\left(\mathbb{R}^{3}\right)} & \lesssim\|f\|_{L^{p}\left(\mathbb{R}^{3}\right)}, \\
\left\|\sum_{J \in \mathbb{Z}^{2}} H_{J}^{\text {mid }} * f\right\|_{L^{p}\left(\mathbb{R}^{3}\right)} & \lesssim\|f\|_{L^{p}\left(\mathbb{R}^{3}\right)} .
\end{aligned}
$$

Set $h(t)=\chi(t) / t$ and $H f(x)=\sum_{J} H_{J}^{1,2} * f(x)$, where

$$
\widehat{H_{J}^{1,2}}(\xi)=\iint e^{i\left[\xi_{1} 2^{j_{1}} t_{1}+\xi_{2} 2^{j_{2}} t_{2}+\xi_{3} \gamma_{1}\left(2^{j_{1}} t_{1}\right) \gamma_{2}\left(2^{j_{2}} t_{2}\right)\right]} h\left(t_{1}\right) h\left(t_{2}\right) d t_{1} d t_{2} .
$$

We also let for each $\nu=1,2$,

$$
\begin{aligned}
& \widehat{H_{J}^{\nu}}(\xi)=\iint e^{i\left[\xi_{\nu} 2^{j_{\nu}} t_{\nu}+\xi_{3} \gamma_{1}\left(2^{j_{1}} t_{1}\right) \gamma_{2}\left(2^{j_{2}} t_{2}\right)\right]} h\left(t_{1}\right) h\left(t_{2}\right) d t_{1} d t_{2}, \\
& \widehat{H_{J}^{0}}(\xi)=\iint e^{i \xi_{3} \gamma_{1}\left(2^{j_{1}} t_{1}\right) \gamma_{2}\left(2^{j_{2}} t_{2}\right)} h\left(t_{1}\right) h\left(t_{2}\right) d t_{1} d t_{2} .
\end{aligned}
$$

We see that by using the dual inequality of (2.10), each of (4.1), (4.2) and (4.3) follows from each of (3.4),(3.5) and (3.8) where $M_{J}^{1,2}$ replaced by $H_{J}^{1,2}$ and $M_{J}^{\nu}$ replaced by $H_{J}^{\nu}$ with $\nu=0,1,2$. For this estimate, we need the following estimates corresponding to (3.2) and (3.7):

Lemma 7. Suppose that $\gamma_{1}$ is an odd function and $\gamma_{2}$ is an even function. If $\sup _{t>0}\left|\frac{\gamma_{2}(2 t)}{\gamma_{2}(t)}\right|$ is finite, then we have the estimate for each $\nu=0,1,2$

$$
\left\|\sum_{J} H_{J}^{\nu} * P_{j_{1}}^{1} * P_{j_{2}}^{2} * f\right\|_{L^{p}\left(\mathbb{R}^{3}\right)} \lesssim\|f\|_{L^{p}\left(\mathbb{R}^{3}\right)},
$$

where (4.4) holds when $P_{j_{1}}^{1}$ and $P_{j_{2}}^{2}$ are replaced by $\left(P_{j_{1}}^{1}\right)^{c}$ and $\left(P_{j_{2}}^{2}\right)^{c}$. 
If both of $\gamma_{1}$ and $\gamma_{2}$ are even, then (4.4) follows immediately from

$$
\widehat{H_{J}^{\nu}}(\xi)=0 \text {. }
$$

Thus the proof of Theorem 2 is completed. Since (4.5) holds for $\nu=0,1$, it suffices to consider $\nu=2$ in Lemma 7 . We define

$$
\widehat{L_{j_{1}, j_{2}}^{3}}(\xi)=\psi\left(\xi_{3} \gamma_{1}\left(2^{j_{1}+3}\right) \gamma_{2}\left(2^{j_{2}}\right)\right)-\psi\left(\xi_{3} \gamma_{1}\left(2^{j_{1}-3}\right) \gamma_{2}\left(2^{j_{2}}\right)\right) .
$$

We observe that

$$
\sum_{n_{2}} \sum_{n_{1}} L_{j_{2}-n_{2}}^{2} * L_{j_{1}-n_{1}, j_{2}}^{3} * f=c f
$$

with some constant $c>0$. In proving Lemma 7 we show that

$$
\left\|\sum_{J} H_{J}^{2} * P_{j_{1}}^{1} * P_{j_{2}}^{2} * L_{j_{2}-n_{2}}^{2} * L_{j_{1}-n_{1}, j_{2}}^{3} * f\right\|_{L^{p}} \lesssim 2^{-c\left(\left|n_{1}\right|+\left|n_{2}\right|\right)}\|f\|_{L^{p}}
$$

Proof of (4.6) for $\boldsymbol{p}=\mathbf{2}$. For any fixed $\xi_{2}$ and $\xi_{3}$, we observe that there exist only finitely many $\left(j_{1}, j_{2}\right)$ 's so that $\widehat{L_{j_{2}-n_{2}}^{2}}(\xi) \widehat{L_{j_{1}-n_{1}, j_{2}}^{3}}(\xi)$ is nonzero. Thus by using this orthogonality combined with Plancherel Theorem, it suffices to prove that for some $c>0$

$$
\left|\widehat{H_{J}^{2}}(\xi) \widehat{L_{j_{2}-n_{2}}^{2}}(\xi) \widehat{L_{j_{1}-n_{1}, j_{2}}^{3}}(\xi)\right| \lesssim 2^{-c\left(\left|n_{1}\right|+\left|n_{2}\right|\right)}
$$

Let us rewrite

$$
H_{J}^{2}(\xi)=\iint e^{i\left[\xi_{2} 2^{j_{2}} t_{2}+\xi_{3} \gamma_{1}\left(2^{j_{1}} t_{1}\right) \gamma_{2}\left(2^{j_{2}} t_{2}\right)\right]} h\left(t_{1}\right) h\left(t_{2}\right) d t_{1} d t_{2} .
$$

In showing (4.7), we prove the following estimates

$$
\begin{aligned}
&\left|H_{J}^{2}(\xi)\right| \lesssim \min \left\{\left|\xi_{2} 2^{j_{2}}\right|^{-1 / 2},\left|\xi_{2} 2^{j_{2}}\right|\right\} \\
&\left|H_{J}^{2}(\xi)\right| \lesssim \min \left\{\left|\xi_{3} \gamma_{1}\left(2^{j_{1}-1}\right) \gamma_{2}\left(2^{j_{2}-1}\right)\right|^{-1},\left|\xi_{3} \gamma_{1}\left(2^{j_{1}}\right) \gamma_{2}\left(2^{j_{2}}\right)\right|\right\}
\end{aligned}
$$

which implies (4.7). For this we need to note that $\left|\xi_{3} \gamma_{1}\left(2^{j_{1}-1}\right) \gamma_{2}\left(2^{j_{2}-1}\right)\right|^{-1}$ is majorized by $\left|\xi_{3} \gamma_{1}\left(2^{j_{1}-1}\right) \gamma_{2}\left(2^{j_{2}}\right)\right|^{-1}$ since $\left|\frac{\gamma_{2}\left(2^{j_{2}}\right)}{\gamma_{2}\left(2^{j_{2}-1}\right)}\right|$ is uniformly bounded.

Now we prove (4.8) and (4.9).

Proof of (4.8). By using the mean value theorem and the cancellation property such as

$$
\iint e^{i\left[\xi_{3} \gamma\left(2^{j_{1}} t_{1}\right) \gamma_{2}\left(2^{j_{2}} t_{2}\right)\right]} h\left(t_{1}\right) h\left(t_{2}\right) d t_{1} d t_{2}=0
$$


we obtain that

$\left|\iint\left(e^{i\left[\xi_{2} 2^{j_{2}} t_{2}+\xi_{3} \gamma_{1}\left(2^{j_{1}} t_{1}\right) \gamma_{2}\left(2^{j_{2}} t_{2}\right)\right]}-e^{i\left[\xi_{3} \gamma\left(2^{j_{1}} t_{1}\right) \gamma_{2}\left(2^{j_{2}} t_{2}\right)\right]}\right) h\left(t_{1}\right) h\left(t_{2}\right) d t_{1} d t_{2}\right| \lesssim\left|\xi_{2} 2^{j_{2}}\right|$

By (2.9),

$$
\left|H_{J}^{2}(\xi)\right| \lesssim\left|\xi_{2} 2^{j_{2}}\right|^{-1 / 2}
$$

Thus (4.8) has been proved.

Proof of (4.9). We apply the mean value theorem and the cancelation property such as

$$
\iint e^{i \xi_{2} 2^{j_{2}} t_{2}} h\left(t_{1}\right) h\left(t_{2}\right) d t_{1} d t_{2}=0
$$

to obtain that

$$
\begin{aligned}
\mid \iint\left(e^{i\left[\xi_{2} 2^{j_{2}} t_{2}+\xi_{3} \gamma_{1}\left(2^{j_{1}} t_{1}\right) \gamma_{2}\left(2^{j_{2}} t_{2}\right)\right]}-e^{i \xi_{2} 2^{j_{2}} t_{2}}\right) & h\left(t_{1}\right) h\left(t_{2}\right) d t_{1} d t_{2} \mid \\
& \lesssim\left|\xi_{3} \gamma_{1}\left(2^{j_{1}}\right) \gamma_{2}\left(2^{j_{2}}\right)\right| .
\end{aligned}
$$

We use integration by parts with respect to $t_{1}$ variable, then by the monotonicity of $t_{1} \mapsto \gamma_{1}^{\prime}\left(2^{j_{1}} t_{1}\right) \gamma_{2}\left(2^{j_{2}} t_{2}\right)$, we obtain the desired bound

$$
\left|\int e^{i\left[\xi_{2} 2^{j_{2}} t_{2}+\xi_{3} \gamma_{1}\left(2^{j_{1}} t_{1}\right) \gamma_{2}\left(2^{j_{2}} t_{2}\right)\right]} h\left(t_{1}\right) d t_{1}\right| \lesssim \frac{1}{\left|\xi_{3} 2^{j_{1}} \gamma_{1}^{\prime}\left(2^{j_{1}-1}\right) \gamma_{2}\left(2^{j_{2}-1}\right)\right|} .
$$

Thus (4.9) follows from the above two inequalities.

Proof of (4.6) for $\boldsymbol{p} \neq \mathbf{2}$. We observe that there is the Littlewood-Paley inequality

$$
\left\|\left(\sum_{J}\left|L_{j_{2}-n_{2}}^{2} * L_{j_{1}-n_{1}, j_{2}}^{3} * f\right|^{2}\right)^{1 / 2}\right\|_{L^{p}} \lesssim\|f\|_{L^{p}},
$$

which follows by the multi-parameter Marcinkiewicz multiplier theorem in the same way as in the proof of (2.10) in Lemma 5. By using (4.10) and its dual inequality, we have

$$
\begin{aligned}
& \left\|\sum_{J} H_{J}^{2} * P_{j_{1}}^{1} * P_{j_{2}}^{2} * L_{j_{2}-n_{2}}^{2} * L_{j_{1}-n_{1}, j_{2}}^{3} * f\right\|_{L^{p}} \\
& \quad \lesssim\left\|\left(\sum_{J}\left|H_{J}^{2} * P_{j_{1}}^{1} * P_{j_{2}}^{2} * L_{j_{2}-n_{2}}^{2} * L_{j_{1}-n_{1}, j_{2}}^{3} * f\right|^{2}\right)^{1 / 2}\right\|_{L^{p}} \\
& \quad \lesssim\left\|\left(\sum_{J}\left|P_{j_{1}}^{1} * P_{j_{2}}^{2} * L_{j_{2}-n_{2}}^{2} * L_{j_{1}-n_{1}, j_{2}}^{3} * f\right|^{2}\right)^{1 / 2}\right\|_{L^{p}} \lesssim\|f\|_{L^{p}}
\end{aligned}
$$

where the second inequality follows from Theorem 1 and Lemma 6 and the last one follows from (4.10) combined with (2.12). 


\section{General surface in higher dimensional space}

We consider the $n$ dimensional surface in $\mathbb{R}^{n+1}$, which is parametrized by

$$
\left\{(t, \Gamma(t)): t=\left(t_{1}, \ldots, t_{n}\right) \in \mathbb{R}^{n}\right\}
$$

where $\Gamma$ need not be a tensor product form. In this section we study the $L^{p}$ boundedness of multi-parameter maximal operator and singular integrals associated with this surface.

\subsection{Maximal operator}

We define a multi-parameter maximal function associated with $\Gamma$ by

$$
\mathcal{M}_{\Gamma}^{n} f\left(x, x_{n+1}\right)=\sup _{r_{1}, \ldots, r_{n}>0} \frac{1}{\prod_{i=1}^{n} r_{i}} \int_{\otimes_{i=1}^{n}\left[0, r_{i}\right]}\left|f\left(x-t, x_{n+1}-\Gamma(t)\right)\right| d t
$$

where $x=\left(x_{1}, \ldots, x_{n}\right)$. We are interested in finding an appropriate condition of $\Gamma$ which makes the operator $\mathcal{M}_{\Gamma}^{n}$ bounded in $L^{p}\left(\mathbb{R}^{n+1}\right)$. In order to establish geometric factors that determine the $L^{p}$ boundedness, we need to investigate the proofs of Theorems 1 through 3 . They are based on the multi-dimensional Van der Corput type Lemma in the high frequency part, and the reduction to the strong maximal operator in the low frequency part. We first describe the curvature condition called infinitesimal doubling which is used for the high frequency estimates. A function $g:[0, \infty) \rightarrow[0, \infty)$ is infinitesimal doubling if there is $\epsilon>0$ such that

$$
g^{\prime}(t)>\epsilon \frac{g(t)}{t} \quad \text { for all } t>0 .
$$

We can observe that the proof of Lemma 1 is based on (2.2) and (2.6). These inequalities in the general form $\Gamma$ can be rewritten as for some $\epsilon>0$,

$$
\begin{aligned}
& \left|\frac{\partial \Gamma_{1}^{\prime}\left(t_{1}, t_{2}\right)}{\partial t_{2}}\right| \geq \epsilon\left|\frac{\Gamma_{1}^{\prime}\left(t_{1}, t_{2}\right)}{t_{2}}\right|, \\
& \left|\frac{\partial \Gamma_{2}^{\prime}\left(t_{1}, t_{2}\right)}{\partial t_{1}}\right| \geq \epsilon\left|\frac{\Gamma_{2}^{\prime}\left(t_{1}, t_{2}\right)}{t_{1}}\right| \text { for all } t>0 .
\end{aligned}
$$

Thus in higher dimensional situation it is natural to assume that for each $k=1, \cdots, n$, there exists at least one $\ell \in\{1, \ldots, n\}$ such that the map $t_{\ell} \rightarrow \Gamma_{k}^{\prime}(t)$ is infinitesimal doubling such that

$$
\exists \epsilon>0 \text { such that }\left|\frac{\partial \Gamma_{k}^{\prime}(t)}{\partial t_{\ell}}\right|>\epsilon\left|\frac{\Gamma_{k}^{\prime}(t)}{t_{\ell}}\right| \forall t .
$$


We next consider the condition of $\Gamma$ in order to treat the low frequency part. In view of (3.3), we see that a parameter restriction is an essential idea for the local part estimate. Let $D$ be a nonempty subset of $\{1, \ldots, n\}$. To the restricted surface $\left\{(t, \Gamma(t)): t_{\nu}=0, \nu \in D\right\}$ we associate the maximal function

$$
\begin{array}{rl}
\mathcal{M}_{\Gamma}^{n, D} & f\left(x, x_{n+1}\right) \\
\quad & =\sup _{r_{i}>0 \text { with } i \notin D} \frac{1}{\prod_{i \notin D} r_{i}} \int_{\bigotimes_{i \notin D}\left[0, r_{i}\right]}\left|f\left(x-\hat{t}_{D}, x_{n+1}-\Gamma\left(\hat{t}_{D}\right)\right)\right| d \hat{t}_{D}
\end{array}
$$

where $\hat{t}_{D}=t-\sum_{\nu \in D}\left\langle t, \mathbf{e}_{\nu}\right\rangle \mathbf{e}_{\nu}$. Since $\Gamma$ is not of tensor product, we see that $\Gamma\left(\hat{t}_{D}\right)$ does not identically vanish in general. This implies that $\mathcal{S}_{\Gamma}^{n, D}$ in Lemma 3 is not reduced to the strong maximal function as in Lemma 4. Instead, $\mathcal{S}_{\Gamma}^{n, D}$ is controlled by $\mathcal{M}_{\Gamma}^{n, D}$ as we have seen in Lemma 3. Thus we need to assume that $\mathcal{M}_{\Gamma}^{n, D}$ is bounded in $L^{p}\left(\mathbb{R}^{n+1}\right)$ for each $D \neq \varnothing$. Now we state the theorem for maximal functions.

Theorem 4. Suppose that $t_{k} \mapsto \Gamma(t)$ is a convex increasing function on $[0, \infty)$ for each fixed $\hat{t}_{k}$ with $k=1, \ldots, n$. In particular, for each $k=1, \ldots, n$, there exists $\ell \in\{1, \ldots, n\}$ such that $t_{\ell} \mapsto \Gamma_{k}^{\prime}$ is infinitesimal doubling in (5.2). Then the $L^{p}\left(\mathbb{R}^{n+1}\right)$ boundedness of $\mathcal{M}_{\Gamma}^{n, D}$ for each $D \neq \varnothing$ implies the $L^{p}\left(\mathbb{R}^{n+1}\right)$ boundedness of $\mathcal{M}_{\Gamma}^{n}$ for $1<p \leq \infty$.

By inductive application of Theorem 4 and the fact that $\gamma^{\prime}$ infinitesimal doubling implies $h$ (where $h(t)=\gamma^{\prime}(t) t-\gamma(t)$ ) infinitesimal doubling in [1], we obtain that

Corollary 1. Suppose that $t_{k} \mapsto \Gamma(t)$ is a convex increasing function on $[0, \infty)$ for each fixed $\hat{t}_{k}$ with $k=1, \ldots, n$. If $t_{k} \mapsto \Gamma_{k}^{\prime}$ is infinitesimal doubling for $k=1, \ldots, n$. Then $\mathcal{M}_{\Gamma}^{n}$ is bounded in $L^{p}\left(\mathbb{R}^{n+1}\right)$ for $1<p \leq \infty$.

Examples. If $g$ is convex increasing function such that $g(0)=0$, then the condition (5.1) is satisfied with $\epsilon=1$. Thus it is enough to check that

- the map $t_{k} \mapsto \Gamma_{k}^{\prime}(t)$ is convex increasing,

- $\left.\Gamma_{k}^{\prime}(t)\right|_{t_{k}=0}=0$,

for the $L^{p}\left(\mathbb{R}^{n+1}\right)$ boundedness of the maximal operator $\mathcal{M}_{\Gamma}^{n}$ by Corollary 1 . We easily see that these conditions are satisfied for the surfaces associated with the following functions $\Gamma$ :

1. for a polynomial with nonnegative coefficients $\mathcal{P}$

$$
\Gamma\left(t_{1}, \ldots, t_{n}\right)=\mathcal{P}\left(e^{-\frac{1}{t_{1}}}, \ldots, e^{-\frac{1}{t_{n}}}\right),
$$


2. for a finite subset $\mathcal{S}$ of the set of all monomials with positive coefficients

$$
\Gamma\left(t_{1}, \ldots, t_{n}\right)=\sum_{\mathcal{Q} \in \mathcal{S}} e^{-\frac{1}{\mathcal{Q}\left(t_{1}, \ldots, t_{n}\right)}}
$$

If $\Gamma(t)$ is defined as a tensor product form $\prod_{i=1}^{n} \gamma_{i}\left(t_{i}\right)$, we can easily check the hypotheses of Theorem 4 hold under the only convexity assumption of $\gamma_{i}$ and $\gamma_{i}(0)=\gamma_{i}^{\prime}(0)=0$ for each $i=1, \ldots, n$.

Corollary 2. Suppose that $\Gamma(t)=\prod_{i=1}^{n} \gamma_{i}\left(t_{i}\right)$. If each $\gamma_{i}$ is a convex function with $\gamma_{i}(0)=\gamma_{i}^{\prime}(0)=0$, then $\mathcal{M}_{\Gamma}^{n}$ is bounded in $L^{p}\left(\mathbb{R}^{n+1}\right)$ for $1<p \leq \infty$.

\subsection{Multiple Hilbert transform}

Let the map

$$
t_{k} \mapsto \Gamma\left(t_{1}, \ldots, t_{k}, \ldots, t_{n}\right)
$$

be extended as an odd or even function in $(-\infty, \infty)$ for each fixed $\hat{t}_{k}$ with $k=$ $1, \ldots, n$. Then we define a multiple Hilbert transform associated with $\Gamma$ as

$$
\mathcal{H}_{\Gamma}^{n} f\left(x, x_{n+1}\right)=p . v . \int \cdots \int f\left(x-t, x_{n+1}-\Gamma(t)\right) \frac{d t_{1}}{t_{1}} \cdots \frac{d t_{n}}{t_{n}} .
$$

Let us state the $L^{p}$ result of $\mathcal{H}_{\Gamma}^{n}$. Suppose that $t_{k} \mapsto \Gamma(t)$ is a convex increasing function on $[0, \infty)$ for each fixed $\hat{t}_{k}$ with $k=1, \ldots, n$. In particular, for each $k=1, \ldots, n$, there exists $\ell \in\{1, \ldots, n\}$ such that $t_{\ell} \mapsto \Gamma_{k}^{\prime}$ is infinitesimal doubling.

Theorem 5. If $t_{k} \mapsto \Gamma(t)$ is an even function for each $k=1, \ldots, n$, then $\mathcal{H}_{\Gamma}^{n}$ is bounded on $L^{p}\left(\mathbb{R}^{n+1}\right)$ for $1<p<\infty$.

Theorem 6. Suppose that $t_{k} \mapsto \Gamma(t)$ is an odd function and $t_{i} \mapsto \Gamma(t)$ is an even function for each $i \neq k$. Then under the condition that

$$
T_{k}=\sup _{t}\left|\frac{\Gamma\left(2 t_{1}, \ldots, 2 t_{k-1}, t_{k}, 2 t_{k+1}, \ldots, 2 t_{n}\right)}{\Gamma\left(t_{1}, \ldots, t_{i}, \ldots, t_{n}\right)}\right|
$$

is bounded, $\mathcal{H}_{\Gamma}^{n}$ is bounded on $L^{p}\left(\mathbb{R}^{n+1}\right)$ for $1<p<\infty$.

Remark 4. It is interesting to weaken the hypothesis of (5.2) by using the appropriate $h$ function of $\Gamma$ and making a general theorem corresponding to curve theory in $[1,8]$. It is known in [2] that the double Hilbert transform corresponding to $\left(t_{1}, t_{2}, t_{1} t_{2}^{2}+\gamma\left(t_{2}\right)\right)$ with odd $\gamma$ is not bounded in $L^{2}\left(\mathbb{R}^{3}\right)$ unless $\gamma$ does not satisfy $h$-doubling condition. 


\section{Proof of Theorem 4}

\subsection{Higher dimensional Van der Corput type lemma}

Let $\Omega_{n}=\{1, \ldots, n\}$. For $A=\left\{\nu_{1}, \ldots, \nu_{k}\right\} \subset \Omega_{n}$ and $J=\left(j_{1}, \ldots, j_{n}\right) \in \mathbb{Z}^{n}$, we define a measure $M_{J}^{A}$ by using the Fourier transform

$$
\widehat{M_{J}^{A}}(\xi)=\int e^{i\left[\xi_{\nu_{1}} 2^{j_{\nu_{1}}} t_{\nu_{1}}+\cdots+\xi_{\nu_{k}} 2^{j_{\nu_{k}}} t_{\nu_{k}}+\xi_{n+1} \Gamma\left(2^{j_{1}} t_{1}, \ldots, 2^{j_{n}} t_{n}\right)\right]} \varphi(t) d t
$$

where we abuse the notation to write $\varphi(t)=\varphi\left(t_{1}\right) \cdots \varphi\left(t_{n}\right)$ for simplicity. In order to obtain the $L^{p}$ boundedness for $\mathcal{M}_{\Gamma}^{n}$ in Theorem 4, it suffices to deal with the maximal function defined by $\sup _{J \in \mathbb{Z}^{n}}\left|M_{J}^{\Omega_{n}} * f\right|$. In controlling the high frequency parts we need the following decay estimate.

Lemma 8. Under the same hypothesis of Theorem 4, we have for $A=$ $\left\{\nu_{1}, \ldots, \nu_{k}\right\} \subset \Omega_{n}$,

$$
\left|\widehat{M_{J}^{A}}(\xi)\right| \lesssim \min \left\{\frac{1}{\left|2^{j_{\nu_{1}}} \xi_{\nu_{1}}\right|^{1 / 2}}, \cdots, \frac{1}{\left|2^{j_{\nu_{k}}} \xi_{\nu_{k}}\right|^{1 / 2}}\right\} .
$$

Proof. Our proof will be just following the proof of Lemma 1 in the general setting. By symmetry it suffices to show that $\left|\widehat{M_{J}^{A}}(\xi)\right| \lesssim\left|2^{j_{\nu_{1}}} \xi_{\nu_{1}}\right|^{-1 / 2}$. Thus we may assume that $\left|2^{j_{\nu_{1}}} \xi_{\nu_{1}}\right|>10^{3}$. We write the phase function

$$
\Psi_{J}(\xi, t)=\xi_{\nu_{1}} 2^{j_{\nu_{1}}} t_{\nu_{1}}+\cdots+\xi_{\nu_{k}} 2^{j_{\nu_{k}}} t_{\nu_{k}}+\xi_{n+1} \Gamma\left(2^{j_{1}} t_{1}, \ldots, 2^{j_{n}} t_{n}\right) .
$$

Fix $J$ and decompose $\widehat{M_{J}^{A}}(\xi)=\sum_{\ell \in \mathbb{Z}} M_{\ell}(\xi)$ such that

$$
M_{\ell}(\xi)=\int e^{i \Psi J(\xi, t)} \chi\left(\frac{2^{j_{\nu_{1}}}\left(\xi_{\nu_{1}}+\xi_{n+1} \Gamma_{\nu_{1}}^{\prime}\left(2^{j_{1}} t_{1}, \ldots, 2^{j_{n}} t_{n}\right)\right)}{2^{\ell}}\right) \varphi(t) d t,
$$

If $2^{\ell}>\left|2^{j_{\nu_{1}}} \xi_{\nu_{1}}\right|^{1 / 2}$, let us apply integration by parts with respect to $t_{\nu_{1}}$ variable, then

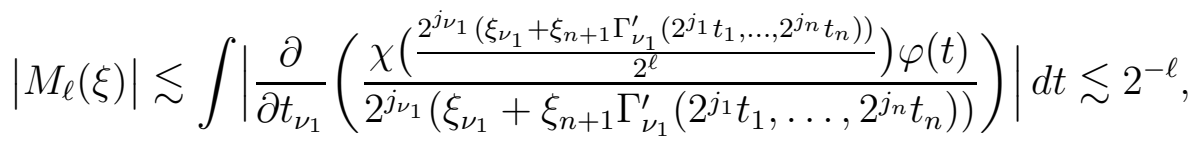

where the second inequality follows by using the monotonicity of the function $t_{\nu_{1}} \mapsto 1 /\left(\xi_{\nu_{1}}+\xi_{n+1} \Gamma_{\nu_{1}}^{\prime}\left(2^{j_{1}} t_{1}, \ldots, 2^{j_{n}} t_{n}\right)\right)$ with each fixed $\hat{t}_{\nu_{1}}$. Thus we see that

$$
\sum_{2^{\ell}>\left|2^{\nu_{1}} \xi_{\nu_{1}}\right|^{1 / 2}}\left|M_{\ell}(\xi)\right| \lesssim\left|2^{j_{\nu_{1}}} \xi_{\nu_{1}}\right|^{-1 / 2}
$$


If $2^{\ell} \leq\left|2^{j_{\nu_{1}}} \xi_{\nu_{1}}\right|^{1 / 2}$, then by the hypothesis of Theorem 4 , there exists $i$ such that

$$
\left|\frac{\partial \Gamma_{\nu_{1}}^{\prime}\left(2^{j_{1}} t_{1}, \ldots, 2^{j_{n}} t_{n}\right)}{\partial t_{i}} 2^{j_{i}} t_{i}\right| \geq \epsilon\left|\Gamma_{\nu_{1}}^{\prime}\left(2^{j_{1}} t_{1}, \ldots, 2^{j_{n}} t_{n}\right)\right| .
$$

By using the support condition such that

$$
\mid 2^{j_{\nu_{1}}}\left(\xi_{\nu_{1}}+\left.\xi_{n+1} \Gamma_{\nu_{1}}^{\prime}\left(2^{j_{1}} t_{1}, \ldots, 2^{j_{n}} t_{n}\right)|<5| 2^{j_{\nu_{1}}} \xi_{\nu_{1}}\right|^{1 / 2}<\left|2^{j_{\nu_{1}}} \xi_{\nu_{1}}\right| / 10\right.
$$

we see that

$$
\left|\xi_{n+1} \Gamma_{\nu_{1}}^{\prime}\left(2^{j_{1}} t_{1}, \ldots, 2^{j_{n}} t_{n}\right)\right| \approx\left|\xi_{\nu_{1}}\right|
$$

From (6.3) and (6.4),

$$
\begin{aligned}
& \left|\xi_{n+1} \frac{\partial \Gamma_{\nu_{1}}^{\prime}\left(2^{j_{1}} t_{1}, \ldots, 2^{j_{n}} t_{n}\right)}{\partial t_{i}} 2^{j_{i}} 2^{j_{\nu_{1}}}\right| \\
& \quad \geq \epsilon\left|\xi_{n+1} \Gamma_{\nu_{1}}^{\prime}\left(2^{j_{1}} t_{1}, \ldots, 2^{j_{n}} t_{n}\right) 2^{j_{\nu_{1}}}\right| \approx\left|2^{j_{\nu_{1}}} \xi_{\nu_{1}}\right| .
\end{aligned}
$$

By using (6.5), we estimate the measure of the integral in $d t_{i}$ with each fixed $\hat{t}_{i}$ to obtain that

$$
\left|\widehat{M}_{\ell}(\xi)\right| \lesssim \int\left|\chi\left(\frac{2^{j_{\nu_{1}}}\left(\xi_{\nu_{1}}+\xi_{n+1} \Gamma_{\nu_{1}}^{\prime}\left(2^{j_{1}} t_{1}, \ldots, 2^{j_{n}} t_{n}\right)\right)}{2^{\ell}}\right) \varphi(t)\right| d t \lesssim \frac{2^{\ell}}{2^{j_{\nu_{1}}}\left|\xi_{\nu_{1}}\right|}
$$

Thus we have

$$
\sum_{2^{\ell} \leq\left|2^{j_{\nu_{1}}} \xi_{\nu_{1}}\right|^{1 / 2}}\left|M_{\ell}(\xi)\right| \lesssim\left|2^{j_{\nu_{1}}} \xi_{\nu_{1}}\right|^{-1 / 2}
$$

Therefore (6.1) follows from (6.2) and (6.6).

\subsection{Proof of Theorem 4}

We decompose

$$
\begin{aligned}
& \widehat{M_{J}^{\Omega_{n}}}(\xi)=\widehat{M_{J}^{\Omega_{n}}}(\xi) \prod_{\nu \in \Omega_{n}}\left(\psi\left(2^{j_{\nu}} \xi_{\nu}\right)+\psi^{c}\left(2^{j_{\nu}} \xi_{\nu}\right)\right) \\
& =\sum_{A \cup B=\Omega_{n},} \widehat{M_{J}^{\Omega_{n}}}(\xi) \prod_{\nu \in A} \psi\left(2^{j_{\nu}} \xi_{\nu}\right) \prod_{\nu \in B} \psi^{c}\left(2^{j_{\nu}} \xi_{\nu}\right) \\
& =\sum_{A \cup B=\Omega_{n}}, \widehat{A \cap B=\varnothing, A \neq \varnothing} \widehat{M_{J}^{A, B}}(\xi),
\end{aligned}
$$


where

$$
\widehat{M_{J}^{A, B}}(\xi)=\widehat{M_{J}^{\Omega_{n}}}(\xi) \prod_{\nu \in A} \psi\left(2^{j_{\nu}} \xi_{\nu}\right) \prod_{\nu \in B} \psi^{c}\left(2^{j_{\nu}} \xi_{\nu}\right)
$$

We write

$$
P_{J}^{A, B}=\left(\circledast_{\nu \in A} P_{j_{\nu}}^{\nu}\right) *\left(\circledast_{\nu \in B}\left(P_{j_{\nu}}^{\nu}\right)^{c}\right)
$$

where we used the notation $\circledast_{k=1}^{n} F_{k}=F_{1} * \cdots * F_{n}$. Then we can write

$$
M_{J}^{A, B}=M_{J}^{\Omega_{n}} * P_{J}^{A, B} .
$$

In order to prove Theorem 4 , we show that for any $A \cup B=\Omega_{n}, A \cap B=\varnothing$ and $A \neq \varnothing$,

$$
\left\|\sup _{J \in \mathbb{Z}^{n}}\left|M_{J}^{A, B} * f\right|\right\|_{L^{p}\left(\mathbb{R}^{n+1}\right)} \lesssim\|f\|_{L^{p}\left(\mathbb{R}^{n+1}\right)} .
$$

Proof. Let us fix a nonempty set $A=\left\{\nu_{1}, \ldots, \nu_{m}\right\}$ and define a measure $N_{J}^{A}$ by

$$
\widehat{N_{J}^{A}}(\xi)=\int e^{i\left[\xi_{n+1} \Gamma\left(2^{j_{1}} t_{1}, \ldots, 2^{j_{n}} t_{n}\right)+\sum_{\nu \in B} \xi_{\nu} 2^{j_{\nu}} t_{\nu}\right]} \prod_{\nu \in A}\left(e^{i \sum_{\nu \in A} \xi_{\nu} 2^{j_{\nu}} t_{\nu}}-1\right) \varphi(t) d t
$$

where $B=\Omega_{n}-A$. Note that

$$
\widehat{N_{J}^{A}}(\xi)=\widehat{M_{J}^{\Omega_{n}}}(\xi)+\sum_{D \in \mathcal{P}(A)} \pm \widehat{M_{J}^{\Omega_{n}-D}}(\xi)
$$

where $\mathcal{P}(A)$ is a family of nonempty subset of $A$. Thus we can write

$$
M_{J}^{A, B}=N_{J}^{A} * P_{J}^{A, B}-\sum_{D \in \mathcal{P}(A)} \pm M_{J}^{\Omega_{n}-D} * P_{J}^{A, B} .
$$

By Lemma 3 and the $L^{p}$ boundedness of $\mathcal{M}_{\Gamma}^{n, D}$ with $D \neq \varnothing$ in the last hypothesis of Theorem 4 , we obtain that for $D \neq \varnothing$

$$
\begin{aligned}
\left\|\sup _{J \in \mathbb{Z}^{n}}\left|M_{J}^{\Omega_{n}-D} * f\right|\right\|_{L^{p}\left(\mathbb{R}^{n+1}\right)} & \approx\left\|\mathcal{S}_{\Gamma}^{n, D} f\right\|_{L^{p}\left(\mathbb{R}^{n+1}\right)} \\
& \lesssim\left\|\mathcal{M}_{\Gamma}^{n, D} f\right\|_{L^{p}\left(\mathbb{R}^{n+1}\right)} \\
& \lesssim\|f\|_{L^{p}\left(\mathbb{R}^{n+1}\right)}
\end{aligned}
$$

Therefore combined with (2.11), we obtain that

$$
\sum_{D \in \mathcal{P}(A)}\left\|\sup _{J \in \mathbb{Z}^{n}}\left|M_{J}^{\Omega_{n}-D} * P_{J}^{A, B} * f\right|\right\|_{L^{p}\left(\mathbb{R}^{n+1}\right)} \lesssim\|f\|_{L^{p}\left(\mathbb{R}^{n+1}\right)}
$$


Thus it suffices to prove that

$$
\left\|\sup _{J \in \mathbb{Z}^{n}}\left|N_{J}^{A} * P_{J}^{A, B} * f\right|\right\|_{L^{p}\left(\mathbb{R}^{n+1}\right)} \lesssim\|f\|_{L^{p}\left(\mathbb{R}^{n+1}\right)} .
$$

We make a dyadic decomposition for the frequency variable $\left(\xi_{1}, \ldots, \xi_{n}\right)$ by using the measure

$$
\mathcal{L}_{j_{1}, \ldots, j_{n}}^{1, \ldots, n}=L_{j_{1}}^{1} * \cdots * L_{j_{n}}^{n} .
$$

In showing (6.12) we prove that

$$
\begin{aligned}
&\left\|\left(\sum_{J}\left|N_{J}^{A} * P_{J}^{A, B} * \mathcal{L}_{j_{1}-\ell_{1}, \ldots, j_{n}-\ell_{n}}^{1, \ldots, n} * f\right|^{2}\right)^{1 / 2}\right\|_{L^{p}\left(\mathbb{R}^{n+1}\right)} \\
& \lesssim 2^{-c\left(\left|\ell_{1}\right|+\cdots+\left|\ell_{n}\right|\right)}\|f\|_{L^{p}\left(\mathbb{R}^{n+1}\right)} .
\end{aligned}
$$

By applying Lemma 7 and the mean value theorem for (6.9) on the support of the frequency parts of $P_{J}^{A, B} * \mathcal{L}_{j_{1}-\ell_{1}, \ldots, j_{n}-\ell_{n}}^{1, \ldots, n}$, we have

$$
\left|\widehat{N_{J}^{A}}(\xi)\right| \lesssim 2^{-c\left(\left|\ell_{1}\right|+\cdots+\left|\ell_{n}\right|\right)}
$$

By (6.15) and the orthogonality of the Littlewood-Paley decomposition of

$$
L_{j_{1}-\ell_{1}}^{1} * \cdots * L_{j_{n}-\ell_{n}}^{n},
$$

we obtain (6.14) with $p=2$. For $p \neq 2$, we can apply the bootstrap argument as in the previous sections.

\section{Proof of Theorem 5 and Theorem 6}

Let $\Omega_{n}=\{1, \ldots, n\}$. For any $A=\left\{\nu_{1}, \ldots, \nu_{k}\right\} \subset \Omega_{n}$, we define a measure $H_{J}^{A}$ so that the Fourier transform $\widehat{H_{J}^{A}}(\xi)$ is given by

$$
\int e^{i\left[\xi_{\nu_{1}} 2^{j \nu_{k}} t_{\nu_{1}}+\cdots+\xi_{\nu_{1}} 2^{j_{\nu_{k}}} t_{\nu_{k}}+\xi_{n+1} \Gamma\left(2^{j_{1}} t_{1}, \ldots, 2^{j} t_{n}\right)\right]} h(t) d t
$$

where we write

$$
h(t)=h\left(t_{1}\right) \cdots h\left(t_{n}\right) .
$$

We repeat the proof of (6.8) by replacing $M_{J}^{A, B}$ by $H_{J}^{A, B}$ and supremum by summation, where

$$
\widehat{H_{J}^{A, B}}(\xi)=\widehat{H_{J}^{\Omega_{n}}}(\xi) \prod_{\nu \in A} \psi\left(2^{j_{\nu}} \xi_{\nu}\right) \prod_{\nu \in B} \psi^{c}\left(2^{j_{\nu}} \xi_{\nu}\right)
$$


It suffices to show that for any $A \cup B=\Omega_{n}, A \cap B=\varnothing$ and $A \neq \varnothing$,

$$
\left\|\sum_{J \in \mathbb{Z}^{n}} H_{J}^{A, B} * f\right\|_{L^{p}\left(\mathbb{R}^{n+1}\right)} \lesssim\|f\|_{L^{p}\left(\mathbb{R}^{n+1}\right)} .
$$

Let us fix the set $A=\left\{\nu_{1}, \ldots, \nu_{m}\right\}$ and $B=\Omega_{n}-A$. We define $R_{J}^{A}$ by

$$
\widehat{R_{J}^{A}}(\xi)=\int e^{i \sum_{\nu \in B} 2^{j_{\nu} \xi_{\nu} t_{\nu}}} \prod_{\nu \in A}\left(e^{i \sum_{\nu \in A} 2^{j_{\nu} \xi_{\nu} t_{\nu}}}-1\right) h(t) d t .
$$

As we have seen in (6.10), we can write

$$
\widehat{R_{J}^{A}}(\xi)=\widehat{H_{J}^{\Omega_{n}}}(\xi)+\sum_{D \in \mathcal{P}(A)} \pm \widehat{H_{J}^{\Omega_{n}-D}}(\xi)
$$

where $\mathcal{P}(A)$ is a family of nonempty subset of $A$. Thus we can write

$$
H_{J}^{A, B}=R_{J}^{A} * P_{J}^{A, B}-\sum_{D \in \mathcal{P}(A)} \pm H_{J}^{\Omega_{n}-D} * P_{J}^{A, B},
$$

where we $P_{J}^{A, B}$ is defined in (6.7). In the same way as (6.12), we obtain that

$$
\left\|\sum_{J \in \mathbb{Z}^{n}} R_{J}^{A} * P_{J}^{A, B} * f\right\|_{L^{p}\left(\mathbb{R}^{n+1}\right)} \lesssim\|f\|_{L^{p}\left(\mathbb{R}^{n+1}\right)}
$$

Thus it suffices to show the corresponding estimate of (6.11) for any $D \neq \varnothing$ :

$$
\left\|\sum_{J} H_{J}^{\Omega_{n}-D} * P_{J}^{A, B} * f\right\|_{L^{p}\left(\mathbb{R}^{n+1}\right)} \lesssim\|f\|_{L^{p}\left(\mathbb{R}^{n+1}\right)} .
$$

Now we prove (7.4) for the rest of our paper. Suppose that the hypothesis of Theorem 5 is true, that is, $t_{\nu} \mapsto \Gamma\left(t_{1}, \ldots, t_{n}\right)$ is an even function for each $\nu=1, \ldots, n$. Then for any $E=\Omega_{n}-D \varsubsetneqq \Omega_{n}$

$$
\widehat{H_{J}^{E}}(\xi)=0 \text {. }
$$

which completes the proof of Theorem 5. Suppose that the hypothesis of Theorem 6 is true. Then without loss of generality we may assume that the map $t_{\nu} \mapsto \Gamma\left(t_{1}, \ldots, t_{n}\right)$ is an even function for each $\nu=2, \ldots, n$, and an odd function for $\nu=1$. We can easily check that if card $(E) \leq n-2$, then (7.5) holds. Thus we consider the case card $(E)=n-1$ only. We can see that (7.5) holds for all $E=\Omega_{n}-\{\nu\}$ with $\nu=2, \ldots, n$. However (7.5) is not true if $E$ is given by $E_{1}=\Omega_{n}-\{1\}=\{2, \ldots, n\}$, that is

$$
\widehat{H_{J}^{E_{1}}}(\xi)=\int e^{i \Phi_{J}^{1}(\xi, t)} h(t) d t
$$


where

$$
\Phi_{J}^{1}(\xi, t)=\xi_{2} 2^{j_{2}} t_{2}+\cdots+\xi_{n} 2^{j_{n}} t_{n}+\xi_{n+1} \Gamma\left(2^{j_{1}} t_{1}, \ldots, 2^{j_{n}} t_{n}\right) .
$$

Thus in order to complete (7.4), it suffices to prove that

Lemma 9. Suppose that the map defined by $t_{\nu} \mapsto \Gamma\left(t_{1}, \ldots, t_{n}\right)$ is an even function with $\nu \neq 1$. If $T_{1}$ in Theorem 6 is finite,

$$
\left\|\sum_{J} H_{J}^{E_{1}} * P_{J}^{A, B} * f\right\|_{L^{p}\left(\mathbb{R}^{n+1}\right)} \lesssim\|f\|_{L^{p}\left(\mathbb{R}^{n+1}\right)} .
$$

\subsection{Proof of Lemma 9}

As in (6.13) we make a dyadic decomposition for the frequency variable $\left(\xi_{2}, \ldots, \xi_{n}\right)$ by using the measure

$$
\mathcal{L}_{j_{2}, \ldots, j_{n}}^{2, \ldots, n}=L_{j_{2}}^{2} * \cdots * L_{j_{n}}^{n}
$$

and make another frequency decomposition for $\xi_{n+1}$ :

$$
\left(\mathcal{L}_{j_{1}, \ldots, j_{n}}^{1}\right)^{\wedge}(\xi)=\psi\left(\xi_{n+1} \Gamma\left(2^{j_{1}+3}, 2^{j_{2}}, \ldots, 2^{j_{n}}\right)\right)-\psi\left(\xi_{n+1} \Gamma\left(2^{j_{1}-3}, 2^{j_{2}}, \ldots, 2^{j_{n}}\right)\right) .
$$

For each fixed $J=\left(j_{1}, \ldots, j_{n}\right)$ and $M=\left(m_{1}, \ldots, m_{n}\right)$ in $\mathbb{Z}^{n}$, we put

$$
\mathcal{L}_{J, M}=\mathcal{L}_{j_{2}-m_{2}, \ldots, j_{n}-m_{n}}^{2, \ldots, n} * \mathcal{L}_{j_{1}-m_{1}, j_{2}, \ldots, j_{n}}^{1} .
$$

Then we see that for each fixed $J \in \mathbb{Z}^{n}$

$$
\sum_{M \in \mathbb{Z}^{n}} \mathcal{L}_{J, M} * f=c f \quad \text { with } \quad c \neq 0
$$

In proving Lemma 9, let us show that

$$
\left\|\sum_{J} H_{J}^{E_{1}} * P_{J}^{A, B} * \mathcal{L}_{J, M} * f\right\|_{L^{p}\left(\mathbb{R}^{n+1}\right)} \lesssim 2^{-c\left(\left|m_{1}\right|+\cdots+\left|m_{n}\right|\right)}\|f\|_{L^{p}\left(\mathbb{R}^{n+1}\right)} .
$$

Proof of (7.6) for $\boldsymbol{p}=\mathbf{2}$. For any fixed $\xi=\left(\xi_{1}, \ldots, \xi_{n+1}\right)$, observe that there exist only finitely many $J=\left(j_{1}, \ldots, j_{n}\right)$ so that $\left(\mathcal{L}_{J, M}\right)^{\wedge}(\xi)$ is nonzero. Thus by using this orthogonality combined with Plancherel Theorem, it suffices to prove

$$
\left|\left(H_{J}^{E_{1}}\right)^{\wedge}(\xi)\left(\mathcal{L}_{J, M}\right)^{\wedge}(\xi)\right| \lesssim 2^{-c\left(\left|m_{1}\right|+\cdots+\left|m_{n}\right|\right)} .
$$


We see that (7.7) follows from the support condition and the estimates:

$$
\begin{aligned}
& \left|\left(H_{J}^{E_{1}}\right)^{\wedge}(\xi)\right| \lesssim \min \left\{\left|\xi_{\nu} 2^{j_{\nu}}\right|^{-1 / 2},\left|\xi_{\nu} 2^{j_{\nu}}\right|: \nu=2, \ldots, n\right\} \\
& \left|\left(H_{J}^{E_{1}}\right)^{\wedge}(\xi)\right| \lesssim \min \left\{\left|\xi_{n+1} \Gamma\left(2^{j_{1}-1}, \ldots, 2^{j_{n}-1}\right)\right|^{-1},\left|\xi_{n+1} \Gamma\left(2^{j_{1}}, \ldots, 2^{j_{n}}\right)\right|\right\} .
\end{aligned}
$$

Note that since $T_{1}$ is finite in the hypothesis of Theorem 6 ,

$$
\left|\xi_{n+1} \Gamma\left(2^{j_{1}-1}, 2^{j_{2}-1} \ldots, 2^{j_{n}-1}\right)\right|^{-1} \lesssim\left|\xi_{n+1} \Gamma\left(2^{j_{1}-1}, 2^{j_{2}} \ldots, 2^{j_{n}}\right)\right|^{-1},
$$

which combined with (7.8) and (7.9) gives the desired bound in (7.7). There remains to show (7.8) and (7.9).

Proof of (7.8). Each $t_{\nu} \mapsto \Gamma(t)$ is an even function for $\nu \neq 1$, so we have

$$
\int e^{i\left[\Phi_{J}^{1}(\xi, t)-2^{j_{\nu}} \xi_{\nu} t_{\nu}\right]} h(t) d t=0
$$

By using (7.10) and the mean value theorem, we obtain that for all $\nu \neq 1$,

$$
\left|\left(H_{J}^{E_{1}}\right)^{\wedge}(\xi)\right|=\left|\int\left(e^{i \Phi_{J}^{1}(\xi, t)}-e^{i\left[\Phi_{J}^{1}(\xi, t)-2^{j_{\nu}} \xi_{\nu} t_{\nu}\right]}\right) h(t) d t\right| \lesssim\left|\xi_{\nu} 2^{j_{\nu}}\right| .
$$

By Lemma 8, we have

$$
\left|H_{J}^{E_{1}}(\xi)\right| \lesssim \min \left\{\left|\xi_{\nu} 2^{j_{\nu}}\right|^{-1 / 2}: \nu=2, \ldots, n\right\} .
$$

Hence (7.8) follows from (7.11) and (7.12).

Proof of (7.9). By using $\int h\left(t_{1}\right) d t_{1}=0$,

$$
\int e^{i\left[\Phi_{J}^{1}(\xi, t)-\xi_{n+1} \Gamma\left(2^{j_{1}} t_{1}, \ldots, 2^{j_{n}} t_{n}\right)\right]} h(t) d t=0 .
$$

Thus by the mean value theorem we obtain that

$$
\begin{aligned}
\mid \int\left(e^{i \Phi_{J}^{1}(\xi, t)}-e^{i\left[\Phi_{J}^{1}(\xi, t)-\xi_{n+1} \Gamma\left(2^{j_{1}} t_{1}, \ldots, 2^{j_{n}} t_{n}\right)\right]}\right) & h(t) d t \mid \\
& \lesssim\left|\xi_{n+1} \Gamma\left(2^{j_{1}}, \ldots, 2^{j_{n}}\right)\right| .
\end{aligned}
$$

Let us apply integration by parts with respect to $t_{1}$ variable, and use the monotonicity of $t_{1} \mapsto \Gamma_{1}^{\prime}\left(2^{j_{1}} t_{1}, \ldots, 2^{j_{n}} t_{n}\right)$. Then we obtain the desired bound

$$
\left|\int e^{i \Phi_{J}^{1}(\xi, t)} h(t) d t\right| \lesssim \frac{1}{\xi_{n+1} 2^{j_{1}} \Gamma_{1}^{\prime}\left(2^{j_{1}-1}, \ldots, 2^{j_{n}-1}\right)} .
$$

Now (7.9) follows from (7.13) and (7.14). 
Proof of (7.6) for $\boldsymbol{p} \neq \mathbf{2}$. As (4.11) we obtain that

$$
\begin{aligned}
\left\|\sum_{J} H_{J}^{E_{1}} * P_{J}^{A, B} * \mathcal{L}_{J, M} * f\right\|_{L^{p}} & \lesssim\left\|\left(\sum_{J}\left|H_{J}^{E_{1}} * P_{J}^{A, B} * \mathcal{L}_{J, M} * f\right|^{2}\right)^{1 / 2}\right\|_{L^{p}} \\
& \lesssim\left\|\left(\sum_{J}\left|P_{J}^{A, B} * \mathcal{L}_{J, M} * f\right|^{2}\right)^{1 / 2}\right\|_{L^{p}} \\
& \lesssim\|f\|_{L^{p}}
\end{aligned}
$$

The first inequality follows from the duality of the last inequality, and the second from Theorem 4 and Lemma 6 . The last inequality follows from (2.12) and the same estimate as in (4.10).

\section{References}

[1] Carbery, A., Christ, M., Vance, J., Wainger, S. and Watson, D.: Operators associated to flat plane curves: $L^{p}$ estimates via dilation methods. Duke Math. J. 59 (1989), no. 3, 675-700.

[2] Carbery, A., Wainger, S. And Wright, J.: Singular integrals and the Newton diagram. Collect. Math. (2006), Vol. Extra, 171-194.

[3] Carbery, A., Wainger, S. and Wright, J.: Hilbert transforms along polynomial surfaces in $\mathbb{R}^{3}$. Duke Math. J. 101 (2000), no. 3, 499-513.

[4] Carlsson, H., Christ, M., Córdoba, A., Douandikoetxea, J., Rubio de Francia, J. L., Vance, J., Wainger, S. and Weinberg, D.: $L^{p}$ estimates for maximal functions and Hilbert transforms along flat curves in $\mathbb{R}^{2}$. Bull. Amer. Math. Soc. (N.S.) 14 (1986), no. 2, 263-267.

[5] Christ, M., Nagel, A., Stein, E. M. And Wainger, S.: Singular and maximal Radon transforms: analysis and geometry. Ann. of Math. (2) 150 (1999), no. 2, 489-577.

[6] Córdoba, A. and Rubio de Francia, J. L.: Estimates for Wainger's singular integrals along curves. Rev. Mat. Iberoamericana 2 (1986), no. 1-2, $105-117$.

[7] Kim, W., Wainger, S., Wright, J. And Ziesler, S.: Singular integrals and maximal functions associated to surfaces of revolution. Bull. London Math. Soc. 28 (1996), no. 3, 291-296.

[8] Nagel, A. Vance, J., Wainger, S. and Weinberg, D.: Hilbert transforms for convex curves. Duke Math. J. 50 (1983), no. 3, 735-744.

[9] Nagel, A. And Wainger, S.: $L^{2}$ boundedness of Hilbert transforms along surfaces and convolution operators homogeneous with respect to a multiple parameter group. Amer. J. Math. 99 (1977), no. 4, 761-785.

[10] Ricci, F. And Stein, E. M.: Multiparameter singular integrals and maximal functions. Ann. Inst. Fourier (Grenoble) 42 (1992), no. 3, 637-670. 
[11] Seeger, A., Wainger, S., Wright, J. and Ziesler, S.: Singular integral and maximal operators associated to hypersurfaces: $L^{p}$ theory. $J$. Geom. Anal. 15 (2005), no. 3, 477-498.

[12] Seeger, A., Wainger, S., Wright, J. and Ziesler, S.: Classes of singular integrals along curves and surfaces. Trans. Amer. Math. Soc. 351 (1999), no. 9, 3757-3769.

[13] Stein, E. M.: Harmonic analysis : real-variable methods, orthogonality, and oscillatory integrals. Princeton Mathematical Series 43. Monographs in Harmonic Analysis, III. Princeton Univ. Press, Princeton, N.Y., 1993.

[14] Wainger, S., Wright, J. And Ziesler, S.: Singular integrals associated to hypersurfaces: $L^{2}$ theory. J. Funct. Anal. 167 (1999), no. 1, 148-182.

Recibido: 8 de mayo de 2007

Yong-Kum Cho

Department of Mathematics

Chung-Ang University

Seoul 156-756, Korea

ykcho@cau.ac.kr

Sunggeum Hong

Department of Mathematics

Chosun University

Gwangju 501-759, Korea

skhong@mail.chosun.ac.kr

Joonil Kim

Department of Mathematics

Yonsei University

Seoul, 120-749, Korea

jikim7030@yonsei.ac.kr

Chan Woo Yang

Department of Mathematics

Korea University

Seoul 136-701, Korea

cw_yang@korea.ac . kr

The third author was supported by the Korea Science and Engineering Foundation(KOSEF) grant funded by the Korea government(MOST) (R01-2007-000-10527-0) and the forth author was supported by the Korea Research Foundation Grant funded by the Korean Government (MOEHRD, Basic Research Promotion Fund) (KRF-2007-331C00012). 